\title{
BESOV SPACES AND THE BOUNDEDNESS OF WEIGHTED BERGMAN PROJECTIONS OVER SYMMETRIC TUBE DOMAINS
}

\author{
Daniele Debertol
}

Abstract
We extend the analysis of weighted Bergman spaces $\mathcal{A}_{\mathbf{s}}^{p, q}$ on sym-
metric tube domains, contained in $[\mathbf{2}]$, to the case where the
weights are positive powers $\Delta_{\mathbf{s}} \doteq \Delta_{1}^{s_{1}-s_{2}} \ldots \cdot \Delta_{r-1}^{s_{r}-s_{r}} \Delta_{r}^{s_{r}}$
of the principal minors $\Delta_{1}, \ldots, \Delta_{r}$ on the symmetric cone $\Omega$. We
discuss the realization of the boundary distributions of functions
in $\mathcal{A}_{\mathbf{s}}^{p, q}$ in terms of Besov-type spaces $B_{\mathbf{s}}^{p, q}$ adapted to the struc-
ture of the cone. We give a necessary and a sufficient condition on
the values of $p, q$ and $\mathbf{s}$ for which this identification between $\mathcal{A}_{\mathbf{s}}^{p, q}$
and $B_{\mathbf{s}}^{p, q}$ holds. We also present a continuous version of these
latter spaces which is new even for the case $s_{1}=\ldots=s_{r}$ con-
sidered in $[\mathbf{2}]$. We use these results to discuss multipliers between
Besov spaces and the boundedness of the weighted Bergman pro-
jection $P_{\mathbf{s}}: L_{\mathbf{s}}^{p, q} \rightarrow \mathcal{A}_{\mathbf{s}}^{p, q}$. The situation in the rank two case is
specifically dealt with.

\section{Introduction}

Let $\Omega$ be an irreducible symmetric cone inside a real vector space $V$ of dimension $n$ endowed with the structure of a euclidean Jordan algebra with identity e. In particular, $\Omega$ is self-dual w.r.t. the inner product

$$
(x \mid y) \doteq \operatorname{tr}(x y)
$$

on $V$. As in [7], we shall define $r \doteq \operatorname{rank}(V)$ and $\Delta_{j}$ to be the $j$-th principal minor on $V, j=1, \ldots, r$. Then, we can write

$$
\Omega=\left\{x \in V: \Delta_{j}(x)>0, \quad j=1, \ldots, r\right\} .
$$

When $\mathbf{s}=\left(s_{1}, \ldots, s_{r}\right) \in \mathbb{R}^{r}$, the generalized power function $\Delta_{\mathbf{s}}$ is defined on $\Omega$ by

$$
\Delta_{\mathbf{s}} \doteq \Delta_{1}^{s_{1}-s_{2}} \cdot \ldots \cdot \Delta_{r-1}^{s_{r-1}-s_{r}} \Delta_{r}^{s_{r}}
$$

2000 Mathematics Subject Classification. 42B35, 32A25.

Key words. Bergman projection, Jordan algebra, Besov multipliers, boundary values. 
We refer to [7], [2] and Section 2.1 below for a more detailed description of these notions.

The family of Bergman spaces $\mathcal{A}_{\mathbf{s}}^{p, q}$ on the tube domain $T_{\Omega} \doteq V+i \Omega$ over the cone $\Omega$ will be defined with respect to the weights $\Delta_{\mathbf{s}}$. The main concern of this paper is the boundedness of the Bergman projection $P_{\mathbf{s}}: L_{\mathbf{s}}^{p, q} \longrightarrow \mathcal{A}_{\mathbf{s}}^{p, q}$. This operator has an explicit kernel given by the formula

$$
K_{\mathbf{s}}(z, w)=d(\mathbf{s}) \Delta_{-\mathbf{s}-\left(\frac{n}{r}, \ldots, \frac{n}{r}\right)}\left(\frac{z-\bar{w}}{i}\right)
$$

for some constant $d(\mathbf{s})$, once $\Delta_{\mathbf{s}}$ functions have been suitably extended to $T_{\Omega}(\operatorname{see}(5.2))$.

The goal of this paper is twofold: first, to develop a continuous analogue of the techniques associated with Besov spaces for the cone, presented in $[\mathbf{2}]$, and second, to generalize some of the topics covered there in the special case $\mathbf{s}=\left(s_{1}, \ldots, s_{r}\right)=(\nu, \ldots, \nu)$ to the wider family of weighted Bergman projections $P_{\mathbf{s}}$, when $\mathbf{s} \neq(\nu, \ldots, \nu)$. In the end, both of these aspects will be seen to rely onto the following remark: the triangular subgroup $T$ in the Iwasawa decomposition $G=T K$ of the structure group $G$ of $\Omega$ is sufficient to perform the analysis of many of the results in $[\mathbf{2}]$. That is, in this paper we show that the $K$ part of $G$ can be made to play no role whatsoever.

In this context, the class of $\Delta_{\mathbf{s}}$ weights is the natural one to be considered: in fact, one can see that generalized powers exhaust the set of continuous and positive homomorphisms of $T$, see [10, 2.4], while group homomorphisms of $G$ must be of the form $g \mapsto \Delta_{r}^{\nu}(g \cdot \mathbf{e})$. Moreover, $\Delta_{\mathrm{s}}$ functions naturally arise in related analytic issues connected to representation theory, see e.g. [7].

We begin with the study of the Bergman spaces $\mathcal{A}_{\mathbf{S}}^{p, q}\left(T_{\Omega}\right)$ associated to the tube domain over $\Omega$ in the complexification of $V, T_{\Omega} \subset V^{\mathbb{C}}$. These are defined to consist of the holomorphic functions $F$ on $T_{\Omega}$ which satisfy the weighted and mixed-norm integrability condition

$$
\|F\|_{L_{\mathbf{s}}^{p, q}} \doteq\left(\int_{\Omega}\left(\int_{V}|F(x+i y)|^{p} d x\right)^{\frac{q}{p}} \Delta_{\mathbf{s}}(y) \frac{d y}{\Delta(y)^{\frac{n}{r}}}\right)^{\frac{1}{q}}<+\infty .
$$

We shall show in Theorem 2.15 that $\mathcal{A}_{\mathbf{s}}^{p, q}$ is non-trivial if and only if $s_{j}>(j-1) \frac{\frac{n}{r}-1}{r-1}$ for every $j=1, \ldots, r$. 
The key tool in our analysis of Bergman projections is a continuous version of the Whitney decomposition used in $[\mathbf{3}],[\mathbf{1}],[\mathbf{2}]$, adapted to the geometric-invariant structure of the cone $\Omega$.

To introduce our framework, let $T^{*}$ denote the adjoint group of $T$ and $d \tau$ the left Haar measure on $T^{*}$; further, for $\widehat{\psi} \in C_{c}^{\infty}(\Omega)$ and $\tau \in T^{*}$, define

$$
\psi_{\tau} \doteq \mathcal{F}^{-1}\left(\widehat{\psi} \circ \tau^{-1}\right)
$$

Then, under a normalizing condition on $\psi$, we will show in Proposition 3.2 that for Schwartz functions $f$ with $\widehat{f}$ supported in $\bar{\Omega}$ the following continuous version of the Littlewood-Paley decomposition holds true:

$$
f=\int_{T^{*}} f * \psi_{\tau} d \tau
$$

One is led by (1.3) to consider the following norm on $\mathcal{S}_{\bar{\Omega}} \doteq\{f \in \mathcal{S}(V) \mid$ $\widehat{f}$ is supported in $\bar{\Omega}\}$ :

$$
\|f\|_{B_{\mathbf{s}}^{p, q}} \doteq\left(\int_{T^{*}} \Delta_{\mathbf{s}}\left((\tau \mathbf{e})^{-1}\right)\left\|f * \psi_{\tau}\right\|_{L}^{q}{ }_{(V, d x)} d \tau\right)^{\frac{1}{q}}
$$

and to introduce a family of homogeneous Besov-type spaces $B_{\mathbf{s}}^{p, q}$ as the completion of $\mathcal{S}_{\bar{\Omega}}$ w.r.t. the norm in (1.4).

Note that the norm in (1.4) is defined in such a way that it enjoys the same invariance properties of the $\mathcal{A}_{\mathbf{s}}^{p, q}$ norm under the action of elements of $T$, see (2.4) and (3.17); on the other hand, the normalization chosen for the $\mathbf{s}$ indices is convenient in order to deal with $\mathcal{A}_{\mathbf{s}}^{p, q}$ spaces, but it does not always match the standard notation in the literature, for instance when $n=1$, see e.g. [15].

We want to stress that it is possible to define a discrete version of the Besov spaces $B_{\mathbf{S}}^{p, q}$ as well, following step by step the construction in [2] for the case $\mathbf{s}=(\nu, \ldots, \nu)$. In fact, we shall show that the two versions of Besov spaces coincide, up to equivalent norms. The path we follow is to concentrate on the new presentation of $B_{\mathbf{s}}^{p, q}$ : as a consequence, when the need occurs for results which are clear generalizations of the corresponding statements in [2], we will often quote them without proof, just adding some extra details if appropriate.

The main advantage in the new presentation of $B_{\mathbf{s}}^{p, q}$ lies in the fact that it is best suited to exploit the simply transitive action of $T^{*}$ on $\Omega$, for $T^{*}$ is a group, while generally the Whitney lattice underlying the discrete decomposition given in $[2]$ is not. This will become apparent in the multiplier Theorem 3.17, for instance, and above all in the final part 
of the paper, when limiting arguments based on Corollary 4.7 come into play.

For the reader's convenience, we summarize below some results about Besov spaces scattered through the paper. Most of them are straightforward generalizations of the corresponding results in [2].

Theorem 1.1. Let $\mathbf{s} \in \mathbb{R}^{r}, 1 \leq p, q \leq+\infty$. Then,

1) $B_{\mathbf{S}}^{p, q}$ is a Banach space, independent of the choice of $\psi$, up to equivalent norms.

2) $B_{\mathbf{s}}^{p, q}$ can be identified with the space of equivalence classes of tempered distributions on $V$ with finite seminorm (1.4) and whose Fourier transform is supported in $\bar{\Omega}$, modulo $\mathcal{S}_{\partial \Omega}^{\prime}$.

3) If $1<p, q<+\infty$, the dual space of $B_{\mathbf{s}}^{p, q}$ can be identified with $B_{-\left(q^{\prime}-1\right) \mathbf{s}}^{p^{\prime}, q^{\prime}}$ by means of the usual pairing.

The continuous version of Besov spaces will also allow for a treatment of multipliers between Besov spaces which, apart being highly suited for the functional calculus of box operators $\square^{\mathrm{s}}$, provides a new, unified formulation of this part of the theory.

The remaining part of the paper deals with the boundedness of Bergman projections. That is, we consider the orthogonal projector $P_{\mathbf{s}}: L_{\mathbf{s}}^{2,2} \longrightarrow \mathcal{A}_{\mathbf{s}}^{2,2}$, and we ask for the existence of bounded extensions into $L_{\mathbf{s}}^{p, q}$ spaces.

In the general case of tube domains over symmetric cones, the hint is given by the Paley-Wiener Theorem 2.7 for $\mathcal{A}_{\mathbf{s}}^{2,2}$ : a function $F \in \mathcal{A}_{\mathbf{s}}^{2,2}$ can be written as the Fourier-Laplace transform $F=\mathcal{L} \widehat{g}$ of a unique distribution (actually, a locally square-integrable function) $g \in B_{\mathbf{s}}^{2,2}$. Note that in particular $g \in \mathcal{S}^{\prime}(V)$ is defined on the Bergman-Shilov boundary $V \times\{0\}$ of $T_{\Omega}$.

Therefore, we plan to exploit the Cauchy extension operator $\mathcal{E}=\mathcal{L} \circ \mathcal{F}$ on $\mathcal{S}_{\bar{\Omega}}$. To begin with, we need a restriction in the indices so that $\mathcal{E}$ is well-defined from $B_{\mathbf{s}}^{p, q}$ into $\mathcal{H o l}\left(T_{\Omega}\right)$. As in the case of [2], we will show that for $1<p, q<+\infty$ this can happen if and only if the distribution $\mathcal{F}^{-1}\left(1_{\Omega} e^{-(\mathbf{e} \mid \cdot)}\right)$ belongs to the dual Besov space $\left(B_{\mathbf{s}}^{p, q}\right)^{*} \simeq B_{-\left(q^{\prime}-1\right) \mathbf{s}}^{p^{\prime}, q^{\prime}}$, which is equivalent to say that

$$
q<Q_{\mathbf{s}}(p) \doteq \min _{j=1, \ldots, r} \frac{s_{j}+\frac{d}{2}(r-j)}{\left(\frac{d}{2}(r-j)-\frac{n}{r p}\right)_{+}} .
$$

Under these assumptions on $p, q$ and $\mathbf{s}$, we can prove the next result, which embodies the concept of boundary values. 
Here the first two statements generalize Theorem 1.7 in $[\mathbf{2}]$ to the family of $\Delta_{\mathbf{s}}$ weights. The last part deserves greater relevance, since it does not have a counterpart even when all indices are equal. This result makes a fundamental use of the continuous notation for Besov spaces (see Corollary 4.7 for the proof).

Theorem 1.2. For every $F$ in $\mathcal{A}_{\mathrm{s}}^{p, q}$ there exists a unique distribution $F_{0} \in B_{\mathbf{s}}^{p, q}$ such that $F=\mathcal{E}\left(F_{0}\right)$. Moreover,

1) $\lim _{\Omega \ni y \rightarrow 0} F_{y}=F_{0}$ both in norm of $B_{\mathbf{s}}^{p, q}$ and in $\mathcal{S}^{\prime}(V)$.

2) There exists $C>0$ s.t. $\left\|F_{0}\right\|_{B_{\mathbf{s}}^{p, q}} \leq C\|F\|_{\mathcal{A}_{\mathbf{s}}^{p, q}}$ for every $F \in \mathcal{A}_{\mathbf{s}}^{p, q}$.

3) If $0 \not \equiv \widehat{\psi} \in C_{c}^{\infty}(\Omega)$ is everywhere non-negative, there exists $a(\psi)>0$ s.t.

$$
F_{0}=a(\psi)^{-1} \int_{T^{*}} F_{\tau^{*}-1} \mathbf{e}^{*} \psi_{\tau} d \tau
$$

distributionally, for every $F \in \mathcal{A}_{\mathbf{s}}^{p, q}$.

We can sloppily state Theorem 1.2 by saying that $\mathcal{E}^{-1}: \mathcal{A}_{\mathbf{s}}^{p, q} \rightarrow B_{\mathbf{s}}^{p, q}$ is a one-to-one bounded operator, and we shall show in Corollary 4.7 that it also has a dense image. Therefore, two questions may naturally be raised:

i) When is $\mathcal{E}^{-1}$ an isomorphism?

ii) Is there a choice of $\psi$ s.t. $\mathcal{E}^{-1}$ can be extended to a bounded operator $\omega_{\mathbf{s}}$ defined on all of $L_{\mathbf{s}}^{p, q}$, with $P_{\mathbf{s}}=\mathcal{E} \circ \omega_{\mathbf{s}}$ ?

Note that i) holds iff $\mathcal{E}\left(B_{\mathbf{s}}^{p, q}\right) \subseteq \mathcal{A}_{\mathbf{s}}^{p, q}$ iff $\mathcal{E}: B_{\mathbf{s}}^{p, q} \rightarrow \mathcal{A}_{\mathbf{s}}^{p, q}$ is bounded iff $\mathcal{E}$ is an isomorphism from $B_{\mathbf{s}}^{p, q}$ onto $\mathcal{A}_{\mathbf{s}}^{p, q}$.

Moreover, we shall show that i) is in a sense equivalent to the statement of ii) w.r.t. the dual indices $p^{\prime}, q^{\prime}$, under some additional assumptions.

Indeed, a limiting argument exploiting the arbitrariness of $\psi$ in (1.5) will allow to define $\omega_{\mathbf{s}}$ on the core $B_{\mathbf{s}}^{2,2}$ as the (Hilbert) adjoint of $\mathcal{E}$, so that in Corollary 5.2 we will be able to prove that $\omega_{\mathrm{s}}$ essentially is the (Banach-wise) dual operator of $\mathcal{E}$ for general $p, q$ and $\mathbf{s}$.

Finally, self-adjointness of $P_{\mathbf{s}}$ and the relation

$$
P_{\mathbf{s}}=\mathcal{E} \circ \omega_{\mathbf{s}}
$$


will provide a proof of the following result, where we let

$$
\begin{aligned}
q_{\mathbf{s}}(p) & \doteq \min \left\{p, p^{\prime}\right\} \min _{j=1, \ldots, r}\left(1+\frac{s_{j}-(j-1) \frac{d}{2}}{\frac{d}{2}(r-j)}\right), \\
p_{\mathbf{s}} & \doteq 1+\min _{j=1, \ldots, r} \frac{s_{j}+\frac{n}{r}}{\left((r-j) \frac{d}{2}-s_{j}\right)_{+}} .
\end{aligned}
$$

Theorem 1.3. Let $s_{j}>(j-1) \frac{\frac{n}{r}-1}{r-1}$ for every $j=1, \ldots, r$, and assume that $1<p<p_{\mathbf{s}}, q_{\mathbf{s}}^{\prime}(p)<q<Q_{\mathbf{s}}(p)$. Then, the following properties are equivalent:

1) $P_{\mathbf{s}}$ admits a bounded extension from $L_{\mathbf{s}}^{p, q}$ onto $\mathcal{A}_{\mathbf{s}}^{p, q}$.

2) $\mathcal{E}$ is an isomorphism from $B_{\mathbf{s}}^{p, q}$ onto $\mathcal{A}_{\mathbf{s}}^{p, q}$.

We shall have occasion to discuss the significance of the indices $q_{\mathbf{s}}(p)$ and $p_{\mathbf{s}}$ later, at least in the special case of rank two, that is, for $n$-dimensional forward light cones. About their relation to this and other questions for higher values of $r$ (but in case $\mathbf{s}=(\nu, \ldots, \nu)$ ), corresponding to different levels of difficulty in the original problem, we suggest reading the survey paper $[\mathbf{5}]$.

Theorem 1.3 states quite clearly that the existence of bounded extensions of $P_{\mathbf{s}}$ into $L_{\mathbf{s}}^{p, q}$ is strictly related to the characterization of boundary values for functions in $\mathcal{A}_{\mathbf{s}}^{p, q}$ as distributions in $B_{\mathbf{s}}^{p, q}$. Therefore, we shall investigate necessary and sufficient conditions for i) to hold, i.e., such that the operator $\mathcal{E}$ be an isomorphism from $B_{\mathrm{s}}^{p, q}$ onto $\mathcal{A}_{\mathbf{s}}^{p, q}$.

To this aim, the better contribution to proving positive results comes from Littlewood-Paley inequalities as in Lemma 4.8 of [2] (see the proof of Theorem 4.8), which is where the stronger restriction imposed by $q_{\mathbf{s}}(p)$ is needed.

When $\mathbf{s}$ is constant, this produces sharp results for $1 \leq p \leq 2$, see [3], [2], while more sophisticated techniques must be used for $p>2$ (see Section 5 in $[\mathbf{2}]$ ).

A main difference in this paper is that, when $\mathbf{s}$ is non-constant, the sufficient conditions of Corollary 1.4 below are no longer known to be sharp even for $p=2$ unless $r=2$ (see Corollary 5.9).

Necessary conditions originating from two different ways of producing counterexamples are summarized in Corollary 4.10. Note that the index $\widetilde{q}_{\mathbf{s}}(p)$ occurring there is not smaller than $q_{\mathbf{s}}(p)$ (and generally strictly bigger). 
Conclusions about $P_{\mathbf{s}}$ are gathered below. When $p=q$, some weaker results than those presented here for tubes $T_{\Omega}$ were obtained in [4], but for a more general class of domains.

Corollary 1.4. Let $s_{j}>(j-1) \frac{\frac{n}{r}-1}{r-1}$ for every $j=1, \ldots, r$, and assume that $1<p<p_{\mathbf{s}}, q_{\mathbf{s}}^{\prime}(p)<q<Q_{\mathbf{s}}(p)$. Then, the following facts are true:

1) $P_{\mathbf{S}}$ is bounded on $L_{\mathbf{s}}^{p, q}$ if $q<q_{\mathbf{s}}(p)$.

2) $P_{\mathbf{s}}$ is unbounded on $L_{\mathbf{s}}^{p, q}$ if $p \leq p_{\mathbf{s}}^{\prime}$ or if $q>\widetilde{q}_{\mathbf{s}}(p)$, and even when $q=\widetilde{q}_{\mathbf{s}}(2)$ if $p \geq 2$.

As (5.4) and (5.12) show, the conditions $q<Q_{\mathbf{s}}(p)$ and $p<p_{\mathbf{s}}$ trivially have to hold if $P_{\mathbf{s}}$ is bounded.

Note that $p_{\mathbf{s}}$ plays no role in case $\mathbf{s}=(\nu, \ldots, \nu)$, for then $\nu>\frac{n}{r}-1$ is automatically satisfied, and $p_{\mathbf{s}}=+\infty$ in this case.

This also means that the case studied here encompasses the one treated in [2], at least when $r>2$ : in fact, for the special case of light cones, the authors of $[\mathbf{2}]$ can use recent progress on the cone multiplier problem (see e.g. [14]) to obtain sharp results when $p$ is sufficiently large.

In the final part of the paper we briefly address the situation in the rank two case, pointing to the gap left open between positive and negative results when $s_{1} \neq s_{2}$.

Finally, I want to express my appreciation to Professor Fulvio Ricci for his advice and warm encouragement throughout the preparation of this work. I also wish to thank the referee for many suggestions which helped to improve the presentation of this material.

\section{Preliminaries}

\subsection{Notations and basic facts.}

We introduce the notation and a list of technical results on symmetric cones, mostly taken from [7], [2]. As a guideline, the reader might think of $\Omega$ as the cone of $r \times r$ real and positive-definite symmetric matrices.

- $\Omega$ is an irreducible symmetric cone inside a vector space $V$ with inner product $(\cdot \mid \cdot)$ and real dimension $n$.

- $V$ is endowed with a Jordan algebra structure with identity e such that $(x \mid y)=\operatorname{tr}(x y)$.

- The rank $r$ of $\Omega$ is the cardinality of any Jordan frame of $V$. We fix a Jordan frame $\left(c_{1}, \ldots, c_{r}\right)$ through the rest of the paper. 
- $V=\bigoplus_{1 \leq i \leq j \leq r} V_{i j}$ is the Pierce decomposition associated with the Jordan frame $\left(c_{1}, \ldots, c_{r}\right)$, with $V_{i i}=\operatorname{sp}\left\{c_{i}\right\}$ and $\operatorname{dim}_{\mathbb{R}} V_{i j}=d$ if $i<j$, so that $\frac{n}{r}-1=\frac{d(r-1)}{2}$.

- $\Delta_{1}, \ldots, \Delta_{r}$ are the principal minors of $V$ w.r.t. the fixed Jordan frame. $\Delta_{j}$ is a homogeneous polynomial of degree $j . \Delta_{r} \equiv \Delta$ is the determinant function.

- $\Delta_{\mathbf{S}} \doteq \Delta_{1}^{s_{1}}\left(\Delta_{2} / \Delta_{1}\right)^{s_{2}} \cdot \ldots \cdot\left(\Delta / \Delta_{r-1}\right)^{s_{r}}$ is a generalized power on $\Omega$, for $\mathbf{s} \in \mathbb{R}^{r}$.

- $G$ is the identity component of the group $\{g \in \mathrm{Gl}(V): g(\Omega)=\Omega\}$.

- $G=N A K$ is the Iwasawa decomposition.

- $K=G \cap \mathrm{O}(V)$ is the stabilizer of $\mathbf{e}$ in $G$.

- $T=N A$ is the triangular subgroup associated to the Pierce decomposition of $V$.

- $T$ acts simply transitively on $\Omega$. That is, the map $T \ni t \mapsto t \cdot \mathbf{e} \in \Omega$ is a diffeomorphism (Gauss decomposition).

- If $P$ denotes the quadratic representation of $V$, we have $A=\left\{P_{a} \mid\right.$ $\left.a=\sum_{j=1}^{r} a_{j} c_{j} \in \Omega\right\}$, and

$$
\Delta_{\mathbf{s}}\left(\left(n P_{a}\right) \cdot x\right)=a_{1}^{2 s_{1}} \cdot \ldots \cdot a_{r}^{2 s_{r}} \Delta_{\mathbf{s}}(x), \quad \forall n \in N, P_{a} \in A .
$$

- $\Delta$ is also invariant under $K$. Moreover,

$$
\operatorname{Det} g=\Delta(g \cdot \mathbf{e})^{\frac{n}{r}}, \quad \forall g \in G .
$$

- The $G$-invariant measure for $\Omega$ is given by

$$
\operatorname{meas}(B) \doteq \int_{B} \frac{d y}{\Delta(y)^{\frac{n}{r}}} .
$$

- We say that $\mathbf{s}>\mathbf{t}$ if and only if $\mathbf{s}$ and $\mathbf{t}$ both belong to $\mathbb{R}^{r}$ and they satisfy $s_{j}>t_{j}$ for each $j \in\{1, \ldots, r\}$. Similarly for $\mathbf{s} \geq \mathbf{t}$.

- $\mathbf{g}_{\mathbf{0}}$ is the $r$-tuple whose $j$-th component is $\frac{d}{2}(j-1)=(j-1) \frac{\frac{n}{r}-1}{r-1}$.

- The generalized Gamma function is defined for $\mathbf{s} \in \mathbb{C}^{r}$ and $y \in \Omega$ by

$$
\Gamma_{\Omega}(\mathbf{s} ; y) \doteq \int_{\Omega} e^{-(\xi \mid y)} \Delta_{\mathbf{s}}(\xi) \frac{d \xi}{\Delta(\xi)^{\frac{n}{r}}} .
$$

The integral is absolutely convergent if and only if $\Re e \mathbf{s}>\mathbf{g}_{\mathbf{0}}$, and in this case

$$
\Gamma_{\Omega}(\mathbf{s} ; y)=\Gamma_{\Omega}(\mathbf{s}) \Delta_{\mathbf{s}}\left(y^{-1}\right),
$$

where we let $\Gamma_{\Omega}(\mathbf{s} ; \mathbf{e}) \doteq \Gamma_{\Omega}(\mathbf{s})$. 
- For $y \in \Omega$, we have

$$
\Delta_{\mathbf{s}}\left(y^{-1}\right)=\Delta_{-\mathbf{s}^{*}}^{*}(y),
$$

where $\Delta_{j}^{*}$ denotes the $j$-th principal minor w.r.t. the rotated Jordan frame $\left(c_{r}, \ldots, c_{1}\right)$ and $\mathbf{s}^{*} \doteq\left(s_{r}, \ldots, s_{1}\right)$.

- $d$ is the Riemannian $G$-invariant distance on $\Omega$ whose associated metric agrees with $(\cdot \mid \cdot)$ on the tangent space at $V$ in e. $B(\xi, \delta)$ is the $d$-ball of radius $\delta$ centered at $\xi \in \Omega$.

Finally, if $A, B>0$, we write $A \preceq_{\epsilon} B$ meaning that $A \leq C(\epsilon) B ; A \sim_{\epsilon} B$ stands for both $A \preceq_{\epsilon} B$ and $B \preceq_{\epsilon} A$.

Also, we say that a function $f: \Omega \rightarrow \mathbb{R}_{+}$is locally almost constant if $f(\xi) \sim_{\delta} f(\eta)$ whenever $d(\xi, \eta) \preceq \delta$, so that we can quote the following result:

Lemma 2.1 ([2, 2.4, 2.9]). The principal minors are locally almost constant. The same is true for the functions $(\cdot \mid y)$ on $\Omega$, uniformly for $y \in \bar{\Omega}$.

\subsection{The Bergman spaces $\mathcal{A}_{\mathrm{s}}^{p, q}$.}

We define the tube over $\Omega$ in the complexification $V^{\mathbb{C}}$ of $V$ as follows:

$$
T_{\Omega} \doteq V+i \Omega \subset V^{\mathbb{C}} \text {. }
$$

Definition 2.2. For $p, q \in[1,+\infty]$ and $\mathbf{s} \in \mathbb{R}^{r}$, let $L_{\mathbf{s}}^{p, q}$ denote the (Banach) space of measurable functions on $T_{\Omega}$ such that

$$
\|f\|_{L_{\mathbf{s}}^{p, q}} \doteq\left(\int_{\Omega}\left(\int_{V}|F(x+i y)|^{p} d x\right)^{\frac{q}{p}} \Delta_{\mathbf{s}}(y) \frac{d y}{\Delta(y)^{\frac{n}{r}}}\right)^{\frac{1}{q}}<+\infty,
$$

and define the Bergman space $\mathcal{A}_{\mathbf{s}}^{p, q}$ as the subspace of $L_{\mathbf{s}}^{p, q}$ formed by its holomorphic functions,

$$
\mathcal{A}_{\mathrm{s}}^{p, q} \doteq L_{\mathrm{s}}^{p, q} \cap \mathcal{H} \operatorname{Lol}\left(T_{\Omega}\right) .
$$

Of course, $d x$ and $d y$ are the usual Lebesgue measure on $V$, so that $\Delta(y)^{-\frac{n}{r}} d y$ is the "left Haar measure" on $\Omega$, as already noted in (2.2).

The choice of $\Delta_{\mathbf{s}}$ entails that a particular Jordan frame $\left(c_{1}, \ldots, c_{r}\right)$ has been fixed, once and for all.

Note that $\mathcal{A}_{\mathbf{s}}^{p, \infty}$ is the Hardy space $\mathcal{H}^{p}$ over the tube $T_{\Omega}$, independently of the choice of $\mathbf{s}$.

As a matter of notation, we will name elements of $\mathcal{A}_{\mathbf{s}}^{p, q}$ by capital letters, such as $F$, and by $F_{y}$ their sections in $L^{p}(V, d x)$ at fixed height $y \in \Omega$. 
We will now quote a result from $[\mathbf{3}]$ whose content asserts that in the special case of rank 2 and constant $\mathbf{s}, \mathcal{A}_{\mathbf{s}}^{p, q}$ is a Banach space. The proof can be easily extended to our general situation. It relies on the fact that convergence in the norm of $L_{\mathbf{s}}^{p, q}$ subsumes uniform convergence over compact subsets of $T_{\Omega}$, and on the following homogeneity property of the $\mathcal{A}_{\mathbf{s}}^{p, q}$ norms $\left(t^{\mathbb{C}}\right.$ is the extension of an element $t \in T$ to $V^{\mathbb{C}}$ by linearity):

$$
\left\|F \circ t^{\mathbb{C}}\right\|_{\mathcal{A}_{\mathbf{s}}^{p, q}}=\|F\|_{\mathcal{A}_{\mathbf{s}}^{p, q}}(\operatorname{Det} t)^{-\frac{1}{p}} \Delta_{\mathbf{s}}\left(t^{-1} \mathbf{e}\right)^{\frac{1}{q}} .
$$

Proposition $2.3([3,4.2])$. Let $F$ belong to $\mathcal{A}_{\mathbf{s}}^{p, q}, u \in[p,+\infty]$ and $w \in \Omega$. Then,

$$
\left\|F_{w}\right\|_{L^{u}(V, d x)} \preceq\|F\|_{\mathcal{A}_{\mathbf{s}}^{p, q} \Delta_{\mathbf{S}}(w)^{-\frac{1}{q}} \Delta(w)^{\frac{n}{r}\left(\frac{1}{u}-\frac{1}{p}\right)} .}
$$

In particular, $\mathcal{A}_{\mathbf{s}}^{p, q}$ is a Banach space.

In the next statement we collect for future reference some standard material on Bergman spaces. For the sake of clarity we sketch the proof, which is patterned after the theory of Hardy spaces on tube domains, see e.g. [16, III.5.7].

We shall see later that we can be dispensed with the condition on $\mathbf{s}$.

Proposition 2.4. Fix $F$ in $\mathcal{A}_{\mathbf{s}}^{p, q}$ for some $\mathbf{s} \geq 0$, and let $u$ be in $[p,+\infty]$. Then,

1) For $w \in \Omega$, the function $F^{w}(z) \doteq F(z+i w)$ is in $\mathcal{H}^{u}$, with

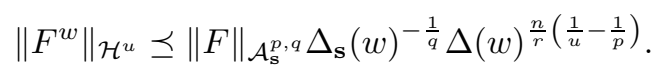

2) For $y \in \Omega, \lim _{|x| \rightarrow+\infty} F(x+i y)=0$, even uniformly as long as $y$ varies over compact subsets of $\Omega$. In particular, $F_{y}$ belongs to $C_{0}(V)$.

3) If $\gg$ denotes the partial order on $V$ induced by $\Omega$, that is,

$$
x \gg x^{\prime} \stackrel{\text { def. }}{\Longleftrightarrow} x-x^{\prime} \in \bar{\Omega},
$$

then the function

$$
y \in(\Omega, \gg) \longmapsto\left\|F_{y}\right\|_{L^{u}(V, d x)}
$$

is decreasing, and moreover $F_{y^{\prime}} \rightarrow F_{y}$ in $L^{u}(V, d x)$ as $y^{\prime} \searrow y$ in $\Omega$ w.r.t. $\gg$.

4) If, moreover, $q<+\infty$, we have that

$$
F=\lim _{\Omega \ni w \rightarrow 0} F^{w}
$$

exists in $\mathcal{A}_{\mathbf{s}}^{p, q}$. In particular, $\mathcal{A}_{\mathbf{s}}^{p, q} \cap \mathcal{H}^{u}$ is dense in $\mathcal{A}_{\mathbf{s}}^{p, q}$, for every $u \in[p,+\infty]$. 
Proof: Since $\Delta_{\mathbf{s}}$ is increasing if $\mathbf{s} \geq 0$, see $[\mathbf{1 0}, 3.8]$, by Proposition 2.3 we have that for every $y \in \Omega$

$$
\begin{aligned}
\left\|\left(F^{w}\right)_{y}\right\|_{L^{u}(V, d x)} & =\left\|F_{w+y}\right\|_{L^{u}(V, d x)} \\
& \preceq\|F\|_{\mathcal{A}_{\mathbf{s}}^{p, q} \Delta_{\mathbf{S}}}(w+y)^{-\frac{1}{q}} \Delta(w+y)^{\frac{n}{r}\left(\frac{1}{u}-\frac{1}{p}\right)} \\
& \preceq\|F\|_{\mathcal{A}_{\mathbf{s}}^{p, q} \Delta_{\mathbf{S}}(w)^{-\frac{1}{q}} \Delta(w)^{\frac{n}{r}\left(\frac{1}{u}-\frac{1}{p}\right)},}
\end{aligned}
$$

and we are done. 2) follows from the mean value theorem for holomorphic functions and (2.4), and finally 3 ) is a direct consequence of 1), 2) and the general theory of Hardy spaces over tubes. 4) is an immediate corollary of 3 ).

\subsection{The Laplace transform and $\mathcal{A}_{\mathrm{s}}^{2, q}$.}

The aim of this section is to prove a simpler characterization of $\mathcal{A}_{\mathbf{s}}^{p, q}$ when $p=2$, in the fashion of classical Paley-Wiener type of results.

We start with recalling the definition of the Laplace transform:

Definition 2.5. For a measurable complex function $g$ on $\Omega$, define $\mathcal{L} g$, the Laplace transform of $g$, as

$$
z \in T_{\Omega} \longmapsto \int_{\Omega} e^{i(z \mid \xi)} g(\xi) d \xi,
$$

whenever this integral is well-defined.

Note that we make use of the sesquilinear extension $(z \mid w)$ to $V^{\mathbb{C}}$ of the real inner product on $V$.

Also note that upon normalizing the inverse Fourier transform on $V$ by

we have that

$$
\left(\mathcal{F}^{-1} g\right)(x) \doteq \int_{V} e^{i(x \mid \xi)} g(\xi) d \xi,
$$

$$
(\mathcal{L} g)_{y}=\mathcal{F}^{-1}\left(e^{-(y \mid \cdot)} g\right)
$$

for every $y \in \Omega$.

The classical Paley-Wiener theorem states that $(2 \pi)^{-n} \mathcal{L}$ is a unitary map of Hilbert spaces between $L^{2}(\Omega, d \xi)$ and $\mathcal{H}^{2}$. Following [3, 7.1], we shall presently see how to suitably modify the domain to obtain $\mathcal{A}_{\mathbf{s}}^{2, q}$ as a range.

Recall the definition of $\mathbf{g}_{\mathbf{0}}$ and its connection with $\Gamma_{\Omega}$ :

$$
\mathbf{g}_{0}=\left(\ldots,(j-1) \frac{d}{2}, \ldots\right) \text { for } j=1, \ldots, r .
$$


Definition 2.6. For $1 \leq q<+\infty$ and $\mathbf{s} \in \mathbb{R}^{r}$ with $\mathbf{s}>\mathbf{g}_{\mathbf{0}}$, let $L_{\mathbf{s}}^{q}(\Omega)$ denote the space of complex, measurable functions $g$ on $\Omega$ such that

$$
\|g\|_{L_{\mathbf{s}}^{q}(\Omega)} \doteq\left(\frac{1}{\Gamma_{\Omega}(\mathbf{s})} \int_{\Omega}\left(\int_{\Omega} e^{-(y \mid \xi)}|g(\xi)|^{2} d \xi\right)^{\frac{q}{2}} \Delta_{\mathbf{s}}(y) \frac{d y}{\Delta(y)^{\frac{n}{r}}}\right)^{\frac{1}{q}}<+\infty .
$$

Notice that we may equivalently replace $y$ by $c y$ for any constant $c>0$ in the definition above, by homogeneity.

Moreover, $L_{\mathbf{s}}^{q}(\Omega)$ clearly is a Banach space, since it can be identified with a closed subspace of a weighted Lebesgue space with mixed norm.

Theorem 2.7. $\left(\frac{2^{s_{1}+\cdots+s_{r}}}{\Gamma_{\Omega}(\mathbf{s})}\right)^{\frac{1}{q}}(2 \pi)^{-n} \mathcal{L}$ is a surjective isometry between $L_{\mathbf{s}}(\Omega)$ and $\mathcal{A}_{\mathbf{s}}^{2, q}$.

Proof: First of all, we verify that $\mathcal{L}$ is well-defined: if $g$ is in $L_{\mathbf{s}}^{q}(\Omega)$, by Lemma 2.1 and invariance we have that for any $w$ in $\Omega$

$$
\begin{aligned}
+\infty>\|g\|_{L_{\mathbf{s}}^{q}(\Omega)}^{q} & \succeq \int_{B(w, 1)}\left(\int_{\Omega} e^{-c(y \mid \xi)}|g(\xi)|^{2} d \xi\right)^{\frac{q}{2}} \Delta_{\mathbf{s}}(y) \frac{d y}{\Delta(y)^{\frac{n}{r}}} \\
& \succeq \Delta_{\mathbf{s}}(w) \int_{B(w, 1)}\left(\int_{\Omega} e^{-(w \mid \xi)}|g(\xi)|^{2} d \xi\right)^{\frac{q}{2}} \frac{d y}{\Delta(y)^{\frac{n}{r}}} \\
& \succeq \Delta_{\mathbf{s}}(w)\left\|e^{-(w \mid \cdot)} g\right\|_{L^{2}(\Omega, d \xi)}^{q},
\end{aligned}
$$

and thus in particular

$$
\left\|e^{-(w \mid \cdot)} g\right\|_{L^{1}(\Omega)} \preceq\left\|e^{-\left(\frac{w}{2} \mid \cdot\right)} g\right\|_{L^{2}(\Omega)} \Delta(w)^{-\frac{n}{2 r}}<+\infty .
$$

Also, (2.7) gives the equality

$$
\|\mathcal{L} g\|_{\mathcal{A}_{\mathbf{s}}^{2, q}}=(2 \pi)^{n}\left(\frac{\Gamma_{\Omega}(\mathbf{s})}{2^{s_{1}+\cdots+s_{r}}}\right)^{\frac{1}{q}}\|g\|_{L_{\mathbf{s}}^{q}(\Omega)}
$$

by the very definition of the norm on $L_{\mathbf{S}}^{q}(\Omega)$. Note that (2.9) and the classical version of the theorem for the Hardy space $\mathcal{H}^{2}$ quoted above imply that the range of $\mathcal{L}$ contains $\mathcal{A}_{\mathrm{s}}^{2, q} \cap \mathcal{H}^{2}$, which is a dense subspace of $\mathcal{A}_{\mathbf{s}}^{2, q}$ by Proposition 2.4.4). Since $L_{\mathbf{s}}^{q}(\Omega)$ is a Banach space, this is sufficient to conclude.

Note that $L_{\mathbf{s}}^{2}(\Omega)=L^{2}\left(\Omega, \Delta_{\mathbf{s}}\left(\xi^{-1}\right) d \xi\right)$ with equal norms, so that in the previous theorem we have a unitary map between Hilbert spaces. 
Also, equation (2.8) is meaningful for $q=+\infty$ : however, $L_{\mathbf{s}}^{\infty}(\Omega)=$ $L^{2}(\Omega, d \xi)$ with equal norms, by Fatou's lemma, and independently of $\mathbf{s}$. Consequently, Theorem 2.7 could have been stated so as to formally include even the classical $\mathcal{H}^{2}$ case.

Corollary 2.8. $\mathcal{A}_{\mathbf{s}}^{2, q} \neq\{0\}$ for $\mathbf{s}>\mathbf{g}_{0}$ and $q<+\infty$.

Proof: Simply note that $1_{B(\mathbf{e}, 1)}$ belongs to $L_{\mathbf{s}}^{q}(\Omega)$ in the stated hypotheses, by Lemma 2.1 .

\subsection{Non-triviality of $\mathcal{A}_{\mathrm{s}}^{p, q}$.}

We shall see later that the condition in Corollary 2.8 is even necessary for the non-triviality of Bergman spaces $\mathcal{A}_{\mathbf{s}}^{p, q}$ for all $p$ 's, if $q<+\infty$.

This is a result which does not seem to appear in the literature in this generality, so we include it in this subsection.

In order to be able to exploit the Laplace transform on some concrete examples, we want to extend $\Delta_{j}$ 's to $V^{\mathbb{C}}$, and this can simply be done by complexifying coordinates, for $\Delta_{j}$ is a homogeneous, real polynomial of degree $j$, see $[7]$.

To quote efficiently the next result, we also define $\Delta_{0} \equiv 1$ on $V^{\mathbb{C}}$.

Lemma 2.9 ([10, 7.3]). Let $z$ belong to $\Omega+i V$ and $j$ to $\{1, \ldots, r\}$. Then, $\Delta_{j}(z) \neq 0$, and the real part of $\frac{\Delta_{j}(z)}{\Delta_{j-1}(z)}$ is strictly positive.

The previous lemma accounts for the problem of choosing the appropriate determination of the argument to enable the definition of generalized powers as holomorphic functions on $-i T_{\Omega}$ : for $\mathbf{s} \in \mathbb{C}^{r}$ and $z \in \Omega+i V$, let

$$
\Delta_{\mathbf{s}}(z) \doteq \Delta_{1}(z)^{s_{1}}\left(\frac{\Delta_{2}(z)}{\Delta_{1}(z)}\right)^{s_{2}} \ldots\left(\frac{\Delta(z)}{\Delta_{r-1}(z)}\right)^{s_{r}},
$$

where we consider the principal branch of log to determine the complex roots.

The new definition of $\Delta_{\mathbf{s}}$ provides for an analytic extension of (1.1), since the two formulas agree on $\Omega$. Actually, we may also show that it gives the analytic extension of (1.1) to $\Omega+i V$, and since we will need similar unicity results frequently later, for convenience sake we recall the basic lemma we make use of:

Lemma 2.10 ([7, IX.1.1]). Let $W$ be a real, finite-dimensional vector space, and $A$ an open subset of $W^{\mathbb{C}}$. Assume that $G$ is a holomorphic function on $A \times(-\bar{A})$, where - denotes conjugation, and that $G(z,-\bar{z})=$ 0 for all $z \in A$. Then, $G$ is identically zero. 
We now have at our disposal all the necessary tools to evaluate the Laplace transform of some specific functions. A careful reading of $[\mathbf{1 1}]$ would reveal that a similar statement, but holding for homogeneous cones, can be found there.

Proposition 2.11. For $\mathbf{t} \in \mathbb{C}^{r}$, let

$$
F_{\mathbf{t}}(\xi) \doteq 1_{\Omega}(\xi) e^{-(\mathbf{e} \mid \xi)} \Delta_{-\mathbf{t}}\left(\xi^{-1}\right) \Delta(\xi)^{-\frac{n}{r}}
$$

Then, $\mathcal{L} F_{\mathbf{t}}$ is pointwise well-defined if and only if $\Re e \mathbf{t}^{*}>\mathbf{g}_{\mathbf{0}}$, with

$$
\left(\mathcal{L} F_{\mathbf{t}}\right)(z)=\Gamma_{\Omega}\left(\mathbf{t}^{*}\right) \Delta_{-\mathbf{t}}(\mathbf{e}-i z) \quad\left(z \in T_{\Omega}\right) .
$$

Moreover, for $\mathbf{s}>\mathbf{g}_{\mathbf{0}}$, we have that $\mathcal{L} F_{\mathbf{t}}$ belongs to $\mathcal{A}_{\mathbf{s}}^{2,2}$ if and only if

$$
2 \Re e \mathbf{t}^{*}>\mathbf{s}^{*}+\mathrm{g}_{0}+\left(\frac{n}{r}, \ldots, \frac{n}{r}\right) .
$$

Proof: The first statement is a direct consequence of (2.3), so that by the above lemma we need only verify equation (2.10) for $z=i y$, with $y \in \Omega$. In this case, the formula follows again by (2.3). The final part of the result is obtained by Theorem 2.7 , since the $L_{\mathbf{s}}^{2}$ norm of $F_{\mathbf{t}}$ can be easily evaluated.

Corollary $2.12([\mathbf{1 0}, 7.8])$. Let $\mathbf{t} \in \mathbb{C}^{r}$ and $y \in \Omega$. Then,

$$
J_{\mathbf{t}}(y) \doteq \int_{V}\left|\Delta_{-\mathbf{t}}(y+i x)\right| d x
$$

converges if and only if $\Re e \mathbf{t}^{*}>\mathbf{g}_{\mathbf{0}}+\left(\frac{n}{r}, \ldots, \frac{n}{r}\right)$, and in this case

$$
J_{\mathbf{t}}(y)=\frac{(2 \pi)^{2 n} 2^{n-\Re e\left(t_{1}+\cdots+t_{r}\right)} \Gamma_{\Omega}\left(\Re e \mathbf{t}^{*}-\left(\frac{n}{r}, \ldots, \frac{n}{r}\right)\right)}{\left|\Gamma_{\Omega}\left(\mathbf{t}^{*} / 2\right)\right|^{2}}\left|\Delta_{-\mathbf{t}}(y)\right| \Delta(y)^{\frac{n}{r}} .
$$

Corollary 2.12 allows one to compute the following integral, whose first evaluation is in [11]:

$$
G_{\mathbf{s}, \mathbf{t}}(u) \doteq \int_{\Omega} \Delta_{\mathbf{t}}(y+u) \Delta_{\mathbf{s}}(y) \frac{d y}{\Delta(y)^{\frac{n}{r}}}
$$

converges absolutely if and only if $\Re e(\mathbf{s}+\mathbf{t})<-\mathbf{g}_{\mathbf{0}}^{*}$ and $\Re e \mathbf{s}>\mathbf{g}_{\mathbf{0}}$. In this case,

$$
G_{\mathbf{s}, \mathbf{t}}(u)=\frac{\Gamma_{\Omega}(\mathbf{s}) \Gamma_{\Omega}\left(-(\mathbf{s}+\mathbf{t})^{*}\right)}{\Gamma_{\Omega}\left(-\mathbf{t}^{*}\right)} \Delta_{\mathbf{s}+\mathbf{t}}(u) \quad(u \in \Omega) .
$$

There still is another peculiar integral that can be explicitly evaluated on $\Omega$, the so-called Beta integral, defined for $\mathbf{s}$ and $\mathbf{t}$ in $\mathbb{C}^{r}$ and $u \in \Omega$ by the formula

$$
B_{\mathbf{s}, \mathbf{t}}(u) \doteq \int_{\Omega \cap(u-\Omega)} \Delta_{\mathbf{t}-\left(\frac{n}{r}, \ldots, \frac{n}{r}\right)}(u-y) \Delta_{\mathbf{s}}(y) \frac{d y}{\Delta(y)^{\frac{n}{r}}} .
$$


It is a well-known result, see e.g. [7, VII.1.7], that the beta integral converges absolutely if and only if both $\Re e \mathbf{s}$ and $\Re e \mathbf{t}$ are strictly bigger than $\mathbf{g}_{\mathbf{0}}$, and in this case

$$
B_{\mathbf{s}, \mathbf{t}}(u)=\frac{\Gamma_{\Omega}(\mathbf{s}) \Gamma_{\Omega}(\mathbf{t})}{\Gamma_{\Omega}(\mathbf{s}+\mathbf{t})} \Delta_{\mathbf{s}+\mathbf{t}}(u) \Delta(u)^{-\frac{n}{r}} .
$$

We can now extend the result in $[\mathbf{3}]$ about non-triviality of Bergman spaces to the present setting. We shall first show the "if" implication.

Proposition 2.13. Let $1 \leq q<+\infty, \mathbf{s} \in \mathbb{R}^{r}$ and assume that $\mathcal{A}_{\mathbf{s}}^{p, q} \neq\{0\}$. Then, $\mathbf{s}>\mathbf{g}_{\mathbf{0}}$.

Proof: We distinguish between two cases: either $\mathbf{s} \geq 0$ or not. Assume first the former condition to hold, and pick $F$ in $\mathcal{A}_{\mathbf{s}}^{p, q} \backslash\{0\}$. Then, $\left\|F_{\mathbf{e}}\right\|_{p}>0$, otherwise by Proposition 2.4.3) we would get that $F \equiv 0$ on $V+i(\mathbf{e}+\Omega)$, and by holomorphicity, $F$ would be identically zero on $T_{\Omega}$, contradicting the initial choice. Thus, applying Proposition 2.4.3) once more, we find that

$$
\begin{aligned}
+\infty>\|F\|_{\mathcal{A}_{\mathbf{s}}^{p, q}}^{q} & =\int_{\Omega}\left\|F_{y}\right\|_{p}^{q} \Delta_{\mathbf{s}}(y) \frac{d y}{\Delta(y)^{\frac{n}{r}}} \\
& \geq\left\|F_{\mathbf{e}}\right\|_{p}^{q} \int_{\Omega \cap(\mathbf{e}-\Omega)} \Delta_{\mathbf{s}}(y) \frac{d y}{\Delta(y)^{\frac{n}{r}}} \\
& =\left\|F_{\mathbf{e}}\right\|_{p}^{q} B_{\mathbf{s},\left(\frac{n}{r}, \ldots, \frac{n}{r}\right)}(\mathbf{e}),
\end{aligned}
$$

and therefore the result above about the beta integral applies. So, only the case with $\mathbf{s} \geq \mathbf{0}$ remains to be considered. For arbitrary $F \in \mathcal{A}_{\mathbf{s}}^{p, q}$, define $G \doteq e^{i(\cdot \mid \mathbf{e})} F$. We claim that that there exists $\mathbf{t} \in \mathbb{R}^{r}$ with $\mathbf{t} \geq \mathbf{0}$ and $\mathbf{t} \ngtr \mathbf{g}_{\mathbf{0}}$ such that $G \in \mathcal{A}_{\mathbf{t}}^{p, q}$. Once this is shown to be true, $\bar{G}$ is identically zero, by the first part of the proof, and consequently $F$ is also. But

$$
\|G\|_{\mathcal{A}_{\mathbf{t}}^{p, q}}^{q}=\int_{\Omega} e^{-q(y \mid \mathbf{e})}\left\|F_{y}\right\|_{p}^{q} \Delta_{\mathbf{t}}(y) \frac{d y}{\Delta(y)^{\frac{n}{r}}},
$$

and

$$
\left\|F_{y}\right\|_{p}^{q} \preceq\|F\|_{\mathcal{A}_{\mathbf{s}}^{p, q}}^{q} \Delta_{-\mathbf{s}}(y)
$$

for every $y \in \Omega$ by $(2.5)$, so that $\|G\|_{\mathcal{A}_{\mathbf{t}}^{p, q}}$ is indeed finite whenever $\mathbf{t}>\mathbf{s}+\mathbf{g}_{\mathbf{0}}$, by (2.3). Now, $\mathbf{s} \geq \mathbf{0}$ means that there exists $j \in\{1, \ldots, r\}$ with $s_{j}<0$ : let $t_{l} \doteq\left|s_{l}\right|+\frac{n}{r}$ if $l \neq j$ and $t_{j} \doteq \frac{d(j-1)}{2}$ to conclude. 
We shall see in a moment that in fact, if $q \neq+\infty$, the condition on $\mathbf{s}$ is also sufficient. A stronger result, which can be adapted from the proof of Corollary 4.5 in $[\mathbf{3}]$, is the following:

Proposition 2.14. Let $\mathbf{s}, \mathbf{t}$ in $\mathbb{R}^{r}$ be strictly bigger than $\mathbf{g}_{\mathbf{0}}$ and $q \neq+\infty$. Then, $\mathcal{A}_{\mathbf{t}}^{u, v} \cap \mathcal{A}_{\mathbf{s}}^{p, q}$ is dense in $\mathcal{A}_{\mathbf{s}}^{p, q}$.

On the other hand, it is also possible to give a direct proof of the characterization of the non-triviality of $\mathcal{A}_{\mathbf{s}}^{p, q}$, a result which already is in [3] for light-cones, with the additional assumption $\mathbf{s}=(\nu, \ldots, \nu)$.

Theorem 2.15. Let $1 \leq q<+\infty, 1 \leq p \leq+\infty$ and $\mathbf{s} \in \mathbb{R}^{r}$. Then, $\mathcal{A}_{\mathbf{s}}^{p, q}$ is non-trivial if and only if $\mathbf{s}>\mathbf{g}_{\mathbf{0}}$.

Proof: The necessary condition has already been proved in Proposition 2.13, while for the sufficiency part it is enough to show that the function $F_{N}(z) \doteq \Delta(\mathbf{e}-i z)^{-N}$ belongs to $\mathcal{A}_{\mathbf{s}}^{p, q}$ if $N$ is chosen sufficiently big. But the inequality

$$
\left\|\left(F_{N}\right)_{y}\right\|_{p} \preceq \Delta(y+\mathbf{e})^{\frac{n}{r_{p}}-N}
$$

holds, by Corollary 2.12 in case $p<+\infty$ and by $[\mathbf{1 0}, 7.5]$ otherwise. Then, the result follows from (2.12) as soon as we choose $N>\frac{2 n}{r}-1$.

Therefore, in the sequel we will be justified in assuming $\mathbf{s}>\mathbf{g}_{\mathbf{0}}$ if necessary, so that all of the previous results hold without any further restriction.

\section{Besov spaces for $\Omega$}

The aim of this part of the paper is to define a continuous version of the Besov-type spaces of distributions adapted to the geometry of the symmetric cone $\Omega$, introduced in $[\mathbf{2}]$. These spaces will play a crucial role later on, concerning the description of boundary values and duals of Bergman spaces, and above all in connection with the boundedness of the Bergman projector. Moreover, the new formulation can be best employed in connection with the study of multipliers between (possibly different) Besov spaces, providing a unified approach to this part of the theory, see e.g. Theorem 3.17.

An important remark about notations: principal minors $\Delta_{k}$ and the triangular group $T$ are defined w.r.t. the same Jordan frame $\left(c_{1}, \ldots, c_{r}\right)$ fixed before. Then, the group $T^{*}$ made of the adjoints of the elements of $T$ is the triangular subgroup w.r.t. the rotated Jordan frame $\left(c_{r}, \ldots, c_{1}\right)$, and we shall tacitly identify $T^{*}$ with $\Omega$ by means of the diffeomorphism

$$
T^{*} \ni \tau \longmapsto \tau \mathbf{e} \in \Omega .
$$




\subsection{Preliminaries.}

Definition 3.1. For a subset $C$ of $V$, we call $\mathcal{S}_{C}$ (respectively, $\mathcal{D}_{C}$ ) the set of Schwartz functions on $V$ whose Fourier transform is supported (respectively, compactly supported) in $C$.

Now, if $\psi$ belongs to $\mathcal{D}_{\Omega}$, define for every $\tau \in T^{*}$

$$
\psi_{\tau} \doteq \frac{1}{(2 \pi)^{n}} \mathcal{F}^{-1}\left(\widehat{\psi} \circ \tau^{-1}\right)
$$

Clearly, we have that the $L^{1}$ norm of $\psi_{\tau}$ does not depend on $\tau$, and we will indifferently denote $\psi_{\tau}$ by $\psi_{\xi}$ if $\xi=\tau \mathbf{e}$.

Also, we will let $d \tau$ stand for the left Haar measure on $T^{*}$ obtained pulling back the measure (2.2) on $\Omega$ by means of the natural identification above.

Then, the continuous decomposition result we alluded to before is the next one:

Proposition 3.2. Assume that

$$
\int_{T^{*}} \widehat{\psi}_{\tau}(\mathbf{e}) d \tau=1
$$

Then, every $f$ in $\mathcal{S}_{\bar{\Omega}}$ has the representation

$$
f=\int_{T^{*}} f * \psi_{\tau} d \tau
$$

as a convergent integral in the Fréchet space $\mathcal{S}(V)$.

Proof: It depends on a lemma giving the appropriate estimates for $f \in$ $\mathcal{S}_{\bar{\Omega}}$, extending non-trivially the analogous result in $[\mathbf{2}, 3.11]$. Since a new set of estimates for principal minors is involved, we will sketch the proof below.

Lemma 3.3. Let $M \in \mathbb{N}, \mathbf{t} \in \mathbb{R}^{r}$ with $t_{1} \geq t_{2} \geq \cdots \geq t_{r} \geq 0$. Then, there exists $L \in \mathbb{N}$, depending on $M$ and $\mathbf{t}$, such that for every $\xi \in \Omega$, $f \in \mathcal{S}_{\bar{\Omega}}$ and $p \in[1,+\infty]$ we have

$$
\left\|f * \psi_{\xi}\right\|_{L} p_{(V, d x)} \preceq_{\mathbf{t}, M} p_{L}(f) \Delta_{\mathbf{t}}^{*}(\xi) \Delta(\xi)^{\frac{n}{r p^{\prime}}}(1+|\xi|)^{-M} .
$$

We are denoting by $p_{L}$ the Schwartz seminorm

$$
p_{L}(\eta) \doteq \sup _{|\alpha| \leq L} \sup _{x \in V}(1+|x|)^{L}\left|\partial^{\alpha} \eta(x)\right|, \quad \eta \in \mathcal{S}(V) .
$$


Proof: The result was proven in [2] for the case $\mathbf{t}=(m, \ldots, m)$, and the (equivalent) inequality

$$
|\widehat{f}(\xi)| \preceq_{m, M} p_{L}(f) \Delta^{m}(\xi)(1+|\xi|)^{-M}
$$

was the fundamental step towards the corresponding estimate (3.3), which was then deduced by a standard argument. So, here we will just point out those few addenda we need to adapt the proof of (3.4) to our situation, i.e., to all stated t's. First of all, we can assume $M=0$, since the full statement follows from this particular case by applying it to $D^{M} f$, if $D$ denotes the Laplacian operator on $V$. Moreover, since $\widehat{f}$ is supported in $\bar{\Omega}$, we have that $\partial^{\alpha} \widehat{f}$ vanishes on $\partial \Omega$, for every $\alpha \in \mathbb{N}^{r}$. Therefore, choosing $l$ as the smallest integer strictly bigger than $\sum_{j=1}^{r} t_{j}$, and letting

$$
\operatorname{dist}(\xi, \partial \Omega) \doteq \inf _{\sigma \in \partial \Omega}|\xi-\sigma| \quad(\xi \in \Omega)
$$

we can approximate $\widehat{f}(\xi)$ with its Taylor polynomial of degree $l$ and find

$$
|\widehat{f}(\xi)| \preceq_{l} p_{l}(\widehat{f}) \min \left\{\operatorname{dist}(\xi, \partial \Omega)^{l}, 1\right\} \max \left\{\operatorname{dist}(\xi, \partial \Omega)^{l-1}, 1\right\} .
$$

Thus, we are done if we prove the estimates

$$
\operatorname{dist}(\xi, \partial \Omega) \leq \Delta_{k}^{*}(\xi)^{\frac{1}{k}}
$$

for every $\xi$ in $\Omega$ and $k$ in $\{1, \ldots, r\}$, which in turn rest upon the following two facts:

1) $\operatorname{dist}\left(P_{a} \mathbf{e}, \partial \Omega\right)=\min _{j} a_{j}^{2}$, if $a=\sum_{j=1}^{r} a_{j} c_{j}$ for strictly positive $a_{j}$ 's.

$2) \operatorname{dist}\left(n^{*} P_{a} \mathbf{e}, \partial \Omega\right) \leq \operatorname{dist}\left(P_{a} \mathbf{e}, \partial \Omega\right)$, for every $n$ in $N$.

Indeed, assume these two facts and write $\xi=n^{*} P_{a} \mathbf{e} \in \Omega$, for some $n \in N$ and $P_{a} \in A$, and let $i$ be such that $a_{i} \leq a_{j}$ for every $j$. Then,

$$
\operatorname{dist}(\xi, \partial \Omega) \leq a_{i}^{2} \leq\left(a_{r-k+1}^{2} \cdots a_{r}^{2}\right)^{\frac{1}{k}}=\Delta_{k}^{*}\left(P_{a} \mathbf{e}\right)^{\frac{1}{k}}=\Delta_{k}^{*}(\xi)^{\frac{1}{k}},
$$

as claimed. So, we show 1$)$ : since $a^{2}-a_{j}^{2} c_{j}$ belongs to $\partial \Omega$, the inequality

$$
\operatorname{dist}\left(P_{a} \mathbf{e}, \partial \Omega\right) \leq\left|a^{2}-\left(a^{2}-a_{j}^{2} c_{j}\right)\right|=a_{j}^{2}
$$

is trivial, for every $j=1, \ldots, r$. On the other hand, for any $\sigma \in \partial \Omega$ there must exist a primitive idempotent $\widetilde{d}$ with $\sigma \widetilde{d}=0$, by spectral decomposition, and consequently

$$
\left|a^{2}-\sigma\right|=\sup \left\{\left|\left(a^{2}-\sigma \mid y\right)\right|:|y| \leq 1\right\} \geq\left(a^{2} \mid \widetilde{d}\right)=\left(L_{a^{2}} \widetilde{d} \mid \widetilde{d}\right) ;
$$

but $L_{a^{2}}$ is a positive operator with eigenvalues in $\left\{\frac{a_{h}^{2}+a_{k}^{2}}{2} \mid h, k=1, \ldots, r\right\}$, so that we have shown $\left|a^{2}-\sigma\right| \geq \min _{j} a_{j}^{2}$. Since this holds for every $\sigma \in \partial \Omega, 1$ ) is done. We finally prove 2 ): by 1 ), it is enough to show that $n^{*} a^{2}-a_{j}^{2} c_{j}$ belongs to $\partial \Omega$ for every $j \in\{1, \ldots, r\}$. Since $A$ normalizes 
$N^{*}$ and $a_{j}^{2} c_{j}=P_{a} c_{j}$, it is even sufficient (and equivalent) to show that $\mathbf{e}-\vartheta c_{j} \in \partial \Omega$ for every $\vartheta \in N^{*}$. But $\vartheta c_{1}=c_{1}$ and $\left(\vartheta c_{j} \mid c_{j}\right) c_{j}=$ $P_{j j}\left(\vartheta c_{j}\right)=\left(P_{j j} \vartheta P_{j j}\right) c_{j}=c_{j}$, by the very definition of $N^{*}$. Therefore,

$$
\mathbf{e}-\vartheta c_{j}=\left(\mathbf{e}-c_{1}\right)+\vartheta\left(c_{1}-c_{j}\right) \in \bar{\Omega}
$$

and

$$
\left(L_{\mathbf{e}-\vartheta c_{j}} c_{j} \mid c_{j}\right)=1-\left(\vartheta c_{j} \mid c_{j}\right)=0,
$$

so that $\mathbf{e}-\vartheta c_{j}$ cannot belong to the open self-dual cone $\Omega$, showing our claim and thus also concluding the proof of the lemma.

Finally, we want to point out that, in effect, a weaker variant of (3.3) will also be useful for us: if $\mathbf{u}$ is in $\mathbb{R}^{r}$ and $u_{1} \geq u_{2} \geq \cdots \geq u_{r} \geq 0$, then

$$
\left\|f * \psi_{\xi}\right\|_{L^{p}(V, d x)} \preceq_{\mathbf{t}, \mathbf{u}} p_{L}(\widehat{f}) \Delta_{\mathbf{t}}^{*}(\xi) \Delta_{-\mathbf{u}}^{*}(\xi+\mathbf{e}) \Delta(\xi)^{\frac{n}{r p^{\prime}}} .
$$

That (3.5) hold may be seen by noting that $|\xi+\mathbf{e}| \leq r(1+|\xi|)$ for $\xi \in \Omega$, and that

$$
\Delta_{k}^{*}(\xi) \preceq|\xi|^{k}
$$

on $\bar{\Omega}$ by homogeneity, for any $k \in\{1, \ldots, r\}$.

Now we can go back to the proof of Proposition 3.2: first of all, notice that

$$
\int_{T^{*}} \widehat{\psi}_{\tau}(\xi) d \tau=1_{\Omega}(\xi)
$$

on $V$, by left invariance of $d \tau$. Therefore, since $\mathcal{F}^{-1}$ is a continuous operator from $\mathcal{S}(V)$ into itself and $\widehat{f} \equiv 0$ outside $\Omega$, it is sufficient to show that $\int_{T^{*}} f * \psi_{\tau} d \tau$ exists as a convergent integral in the Fréchet space $\mathcal{S}(V)$.

This requires an argument about vector-valued integrals, which is new compared to [2]: let us call $B_{l}$ the completion of $\mathcal{S}(V)$ w.r.t. the norm $p_{l}$, for every $l \in \mathbb{N}$. Note that $B_{l}$ is actually made up of concrete functions, and it is separable. Therefore, by Pettis Theorem in [19, V.4], continuity of the map

$$
\tau \in T^{*} \stackrel{F_{l}}{\longmapsto} f * \psi_{\tau} \in B_{l}
$$

is sufficient to perform the Bochner integral $\int_{T^{*}} f * \psi_{\tau} d \tau$ into the Banach space $B_{l}$, provided that

$$
\int_{T^{*}} p_{l}\left(\widehat{f * \psi_{\tau}}\right) d \tau<+\infty
$$


So we are left to show (3.7), for every $l$ in $\mathbb{N}$ : but by Hausdorff-Young inequality, equation (2.1) and Lemma 3.3, we have that

$$
\int_{T^{*}} p_{l}\left(\widehat{f * \psi_{\tau}}\right) d \tau \preceq_{l, m, u} p_{\widetilde{l}}(f) \int_{\Omega} \frac{\Delta(\xi)^{m}}{\Delta(\xi+\mathbf{e})^{u}} \frac{d \xi}{\Delta(\xi)^{\frac{n}{r}}},
$$

which is a finite quantity by (2.12) if $m$ and $u$ are chosen sufficiently large. Thus, $\int_{T^{*}} f * \psi_{\tau} d \tau$ exists in every $B_{l}$, therefore defining an element of $\mathcal{S}(V)$, as claimed.

Remark 3.4. Since the modular function $\Delta_{T^{*}}$ of $T^{*}$ is given by

$$
\Delta_{T^{*}}(\tau)=\Delta_{\mathbf{g}_{\mathbf{0}}^{*}-\mathbf{g}_{\mathbf{0}}}^{*}(\tau \mathbf{e}),
$$

see [7, VI.3.9], we have that condition (3.2) may also be stated as

$$
\int_{\Omega} \widehat{\psi}(\xi)\left(\Delta_{1}^{*}(\xi) \cdots \Delta_{r-1}^{*}(\xi)\right)^{-d} \frac{d \xi}{\Delta(\xi)}=1 .
$$

\subsection{Continuous and discrete descriptions.}

As mentioned in the introduction, we shall consider the following quantity, which will soon be seen to define a norm on $\mathcal{S}_{\bar{\Omega}}$ :

$$
\|f\|_{B_{\mathbf{s}}^{p, q}} \doteq\left(\int_{T^{*}} \Delta_{\mathbf{s}}\left((\tau \mathbf{e})^{-1}\right)\left\|f * \psi_{\tau}\right\|_{L^{p}(V, d x)}^{q} d \tau\right)^{\frac{1}{q}} ;
$$

here $p, q$ belong to $[1,+\infty]$ (with the obvious modification if $q=+\infty$ ), while $\mathbf{s}$ is in $\mathbb{R}^{r}$.

Lemma 3.5. The relative Schwartz topology is finer than the \|\|$_{B_{s}^{p, q-}}$ topology on $\mathcal{S}_{\bar{\Omega}}$.

Proof: We may assume that $q$ is finite, otherwise the result follows from (3.3) and (3.6). With this proviso, by (3.5) we have that

$$
\|f\|_{B_{\mathbf{s}}^{p, q}} \preceq_{\mathbf{t}, \mathbf{u}} p_{L}(f)\left(\int_{T^{*}} \Delta_{q \mathbf{t}-\mathbf{s}^{*}}^{*}(\tau \mathbf{e}) \Delta_{-q \mathbf{u}}^{*}(\tau \mathbf{e}+\mathbf{e}) \Delta(\tau \mathbf{e})^{\frac{n q}{r^{p}}} d \tau\right)^{\frac{1}{q}},
$$

for every $f$ in $\mathcal{S}_{\bar{\Omega}}$. Owing to (2.12), the integral on the right is convergent if $\mathbf{t}$ and $\mathbf{u}$ are chosen (and fixed) sufficiently large.

Note that actually (3.9) defines a norm on $\mathcal{S}_{\bar{\Omega}}$, by continuity of the $\operatorname{map} T^{*} \ni \tau \mapsto f * \psi_{\tau} \in L^{p}(V, d x)$.

Definition 3.6. We define the Besov space $B_{\mathbf{s}}^{p, q}$ as the completion of $\mathcal{S}_{\bar{\Omega}}$ w.r.t. the norm (3.9).

Our intention is now to produce a concrete realization of $B_{\mathbf{S}}^{p, q}$ as a subspace (in fact, a quotient subspace) of distributions with spectrum in $\bar{\Omega}$. 
Definition 3.7. For a closed subset $C$ of $V$, we define $\mathcal{S}_{C}^{\prime}$ as the set of tempered distributions on $V$ whose Fourier transform is supported in $C$.

From now on we adopt the convention to replace any occurrence of $L^{\infty}$ by $C_{0}$.

Definition 3.8. For $p, q \in[1,+\infty]$ and $\mathbf{s} \in \mathbb{R}^{r}$, let $L_{\mathbf{s}}^{p, q}\left(T^{*}\right)$ denote the vector-valued Lebesgue space $L^{q}\left(T^{*}, \Delta_{-\mathbf{s}^{*}}^{*} d \tau\right)$ taking its values in $L^{p}(V, d x)$. Then, we define

$$
\widetilde{B}_{\mathbf{s}}^{p, q} \doteq\left\{S \in \mathcal{S}_{\bar{\Omega}}^{\prime} \mid\left(\tau \longmapsto S * \psi_{\tau}\right) \in L_{\mathbf{s}}^{p, q}\left(T^{*}\right)\right\} / \mathcal{S}_{\partial \Omega}^{\prime} .
$$

As a matter of notation, we let $\langle U, h\rangle$ simply stand for $U(h)$ and $\breve{h}(x) \doteq h(-x)$ for functions $h \in \mathcal{S}(V)$ and distributions $U \in \mathcal{S}^{\prime}(V)$.

We first claim that for $S \in \mathcal{S}_{\bar{\Omega}}^{\prime}$ we have

$$
S * \psi_{\tau}=0 \text { for almost every } \tau \text { in } T^{*} \Longleftrightarrow S \in \mathcal{S}_{\partial \Omega}^{\prime},
$$

where we are still assuming that (3.2) holds (actually, it is even sufficient to assume $\left.\int_{T^{*}} \widehat{\psi}_{\tau}(\mathbf{e}) d \tau \neq 0\right)$. Indeed, if $g$ is a Schwartz function whose support is contained in $\Omega$, by Proposition 3.2 we have that

$$
\langle\widehat{S}, g\rangle=\left\langle\check{S}, \int_{T^{*}} \widehat{\check{g}} * \psi_{\tau} d \tau\right\rangle=\int_{T^{*}}\left\langle S * \psi_{\tau}, \widehat{g}\right\rangle d \tau
$$

Therefore, independently of the representative chosen for $[S]$, we can define

$$
\|[S]\|_{\widetilde{B}_{\mathbf{s}}^{p, q}} \doteq\left\|\left(\tau \longmapsto S * \psi_{\tau}\right)\right\|_{L_{\mathbf{s}}^{p, q}\left(T^{*}\right)} .
$$

Remark 3.9. We show now that any other Schwartz function $\theta$ in $\mathcal{D}_{\Omega}$ satisfying $c(\theta) \doteq \int_{T^{*}} \widehat{\theta}_{\tau}(\mathbf{e}) d \tau \neq 0$ gives raise to an equivalent norm, so that in particular the finiteness of \|\|$_{\widetilde{B}_{\mathrm{s}}^{p, q}}$ does not depend on the function $\psi$ chosen, and consequently $\widetilde{B}_{\mathbf{s}}^{p, q}$ is intrinsecally determined as a set.

Proof: It clearly suffices to prove just a one-sided inequality, that in a somewhat sloppy but hopingly self-explanatory notation we write as

$$
\|\|_{\theta} \preceq\|\|_{\psi} \text {. }
$$

As far as (3.11) is concerned, it is not even necessary to assume that $c(\theta) \neq 0$.

It is easily seen that (3.10) implies that we have the following weak* decomposition for tempered distribution $S$ whose Fourier transform is compactly supported in $\Omega$ :

$$
S=\int_{T^{*}} S * \psi_{\tau} d \tau
$$


Now, assume that the function $\tau \mapsto S * \psi_{\tau}$ is in $L_{\mathbf{s}}^{p, q}\left(T^{*}\right)$ : in particular, for a fixed $\tau_{0} \in T^{*}$ we have

$$
\left\|S * \theta_{\tau_{0}}\right\|_{p} \preceq \int_{B\left(\tau_{0}, 2 R\right)}\left\|S * \psi_{\tau}\right\|_{p} d \tau
$$

if $R$ is chosen in such a way that both supports of $\widehat{\psi}$ and $\widehat{\theta}$ are contained in $B(\mathbf{e}, R)$. Thus, if $q=+\infty$ we conclude easily by left invariance of $d \tau$. Otherwise, we can apply Hölder inequality, and integrating both sides in $\Delta_{-\mathbf{s}^{*}}^{*} d \tau_{0}$ over $T^{*}$ we finally end up with $(3.11)$.

In order to conclude that $\widetilde{B}_{\mathbf{s}}^{p, q}$ is indeed a realization of $B_{\mathbf{s}}^{p, q}$, it is convenient to introduce a discrete version of these Besov spaces, patterned after the presentation given in $[\mathbf{2}]$. We recall the basic facts we need.

The fundamental tool is a covering lemma, the Whitney decomposition of the symmetric cone $\Omega$, in the form given in $[\mathbf{2}, 2.6]$. It asserts that to any fixed $\delta>0$ one can associate a sequence of points $\left(\xi_{j}\right)_{j \in \mathbb{N}}$ and a partition $\left\{E_{j} \mid j \in \mathbb{N}\right\}$ of $\Omega$ in such a way that $B\left(\xi_{j}, \delta / 2\right) \subseteq E_{j} \subseteq B\left(\xi_{j}, \delta\right)$ and that, moreover, the collection of balls $\left\{B\left(\xi_{j}, R\right) \mid j \in \mathbb{N}\right\}$ of any radius $R>0$ has the finite intersection property.

Then, proceeding as in $[\mathbf{2}, 3.2]$, one can construct a family of $C^{\infty}$ functions $\widehat{\chi}_{j}$ subordinated to this covering (with $\delta \doteq 1$, say) whose inverse Fourier transforms are uniformly bounded in $L^{1}(V, d x)$ and such that $\sum_{j \in \mathbb{N}}\left|\widehat{\chi}_{j}\right|^{2}$ is bounded below.

Definition 3.10. Given a distribution $S \in \mathcal{S}^{\prime}(V)$, let

$$
\|S\|_{\ddot{B}_{\mathbf{s}}^{p, q}} \doteq\left(\sum_{j \in \mathbb{N}} \Delta_{\mathbf{s}}\left(\xi_{j}^{-1}\right)\left\|f * \chi_{j}\right\|_{L}^{q}(V, d x)\right)^{\frac{1}{q}},
$$

with the usual modification in case $q=+\infty$. Then, the discrete Besov space $\ddot{B}_{\mathbf{S}}^{p, q}$ is defined as

$$
\ddot{B}_{\mathbf{s}}^{p, q} \doteq\left\{S \in \mathcal{S}_{\bar{\Omega}}^{\prime} \mid\|S\|_{\ddot{B}_{\mathbf{s}}^{p, q}}<+\infty\right\} / \mathcal{S}_{\partial \Omega}^{\prime}
$$

Following closely the footsteps in $[\mathbf{2}]$ and mimicking the proofs therein, one can show without much effort that the statement below holds in this generalized setting, in the full range of $p, q$ and $\mathbf{s}$. 
Proposition $3.11([2,3.25])$. Let $p, q \in[1,+\infty]$ and $\mathbf{s} \in \mathbb{R}^{r}$. Then, $\ddot{B}_{\mathbf{s}}^{p, q}$ is a Banach space, with $\mathcal{D}_{\Omega}$ as a dense subspace. If, in addition, $\sum_{j} \widehat{\chi}_{j}=1_{\Omega}$, then

$$
[S]=\sum_{j \in \mathbb{N}}\left[S * \chi_{j}\right]
$$

for every $[S] \in \ddot{B}_{\mathbf{s}}^{p, q}$.

In the end, to round things off, we are left to prove that the continuous version is equivalent with the discrete one. More precisely, choose any function $\psi \in \mathcal{D}_{\Omega}$ satisfying $c(\psi) \neq 0$, and let $\left(\xi_{j}\right)_{j \in \mathbb{N}}$ be any $\delta$-lattice, $\delta>0$, for which the following condition holds: there exists a strictly positive number $m$ such that

$$
\Phi(\xi) \doteq \sum_{j \in \mathbb{N}}\left|\widehat{\psi}_{\xi_{j}}(\xi)\right|^{2} \geq m
$$

for $\xi \in \Omega$. Then, the claim is that for $S \in \mathcal{S}_{\bar{\Omega}}^{\prime}$ we have

$$
\|[S]\|_{\widetilde{B}_{\mathbf{s}}^{p, q}} \sim_{\psi}\|[S]\|_{\ddot{B}_{\mathbf{s}}^{p, q}},
$$

so that we can finally state:

Proposition 3.12. $\widetilde{B}_{\mathbf{s}}^{p, q}=\ddot{B}_{\mathbf{s}}^{p, q}$.

Indeed, by (3.15) we would deduce that the identity mapping of $\widetilde{B}_{\mathbf{s}}^{p, q}$ into $\ddot{B}_{\mathbf{s}}^{p, q}$ is bicontinuous, and we already know the latter to have a complete norm. Incidentally, (3.15) and (3.11) also show that the choice of different Whitney lattices, even w.r.t. different $\delta$ 's, does not affect the structure of $\ddot{B}_{\mathbf{s}}^{p, q}$ as a topological vector space, but it only leads to equivalent norms.

Proof of (3.15): The inequality in the $\succeq_{\psi}$ direction is easier, and in this case (3.14) plays no role: if $[S]$ is in $\widetilde{B}_{\mathbf{s}}^{p, q},(3.12)$ tells us that $S * \psi_{\xi_{j}}$ is in $L^{p}(V, d x)$ (respectively, in $C_{0}$ if $p=+\infty$ ) for every $j \in \mathbb{N}$, and that for sufficiently big $R$ we have

$$
\left\|S * \psi_{\xi_{j}}\right\|_{p} \preceq \int_{B\left(\xi_{j}, 2 R\right)}\left\|S * \psi_{\tau}\right\|_{p} d \tau .
$$

Therefore, for $q=+\infty$ the result follows trivially from left invariance of $d \tau$. Otherwise, applying Hölder inequality, Lemma 2.1 and left invariance again, we find

$$
\Delta_{\mathbf{s}}\left(\xi_{j}^{-1}\right)\left\|S * \psi_{\xi_{j}}\right\|_{p}^{q} \preceq \int_{B\left(\xi_{j}, 2 R\right)} \Delta_{-\mathbf{s}^{*}}^{*}(\tau \mathbf{e})\left\|S * \psi_{\tau}\right\|_{p}^{q} d \tau .
$$


Summing over $j$, we conclude by the finite intersection property of Whitney lattices.

Now, for the opposite inequality: note that the function $\Phi$ belongs to $C^{\infty}(\Omega)$ and it is bounded below and above, by (3.14) and the finite intersection property of a Whitney lattice. Therefore, letting

$$
N_{\tau} \doteq\left\{k \in \mathbb{N} \mid B\left(\xi_{k}, R\right) \cap B(\tau \mathbf{e}, R) \neq \emptyset\right\}
$$

for $\tau \in T^{*}$, we can write

$$
S * \psi_{\tau}=(2 \pi)^{-n} \sum_{k \in N_{\tau}}\left(S * \psi_{\xi_{k}}\right) * \bar{\psi}_{\xi_{k}} * \mathcal{F}^{-1}\left(\frac{\widehat{\psi}_{\tau}}{\Phi}\right) \in L^{p}(V, d x)
$$

with

$$
\left\|S * \psi_{\tau}\right\|_{p} \preceq \sum_{k \in N_{\tau}}\left\|S * \psi_{\xi_{k}}\right\|_{p} .
$$

Moreover, the sets $N_{\tau}$ are locally almost constant, and as a consequence the function $\tau \in T^{*} \mapsto S * \psi_{\tau}$ is continuous. So, for $q=+\infty$ we conclude easily by (3.16), and otherwise carrying on as usual we find that for $\tau$ in $E_{j}$

$$
\left\|S * \psi_{\tau}\right\|_{p}^{q} \preceq \sum_{k \in N_{j}}\left\|S * \psi_{\xi_{k}}\right\|_{p}^{q} .
$$

Finally, using Lemma 2.1 once more and interchanging a summation order, we obtain

$$
\begin{aligned}
\|[S]\|_{\widetilde{B}_{\mathbf{s}}^{p, q}}^{q} & \preceq \sum_{j \in \mathbb{N}} \Delta_{\mathbf{s}}\left(\xi_{j}^{-1}\right) \int_{E_{j}}\left\|S * \psi_{\tau}\right\|_{p}^{q} d \tau \\
& \preceq \sum_{j \in \mathbb{N}} \sum_{k \in N_{j}} \Delta_{\mathbf{s}}\left(\xi_{k}^{-1}\right)\left\|S * \psi_{\xi_{k}}\right\|_{p}^{q} \preceq\|[S]\|_{\ddot{B}_{\mathbf{s}}^{p, q}}^{q} .
\end{aligned}
$$

\subsection{Invariance and duality.}

Therefore, from now on we can and shall feel free not to distinguish between these two equivalent realizations of the Besov space $B_{\mathbf{s}}^{p, q}$, even if we will usually adopt the continuous version as being the most convenient one. For instance, the proposition below, which is in $[\mathbf{2}, 3.8]$ for the discrete setting, becomes trivial here. Note that, consistently with the idea to represent Bergman spaces by means of Besov spaces, the norm (3.9) had to be defined that way, and w.r.t. $T^{*}$, in order to match exactly the same homogeneity relation w.r.t. $T$ shown in (2.4). 
Proposition 3.13. $B_{\mathbf{s}}^{p, q}$ is invariant for the action of $T$, and

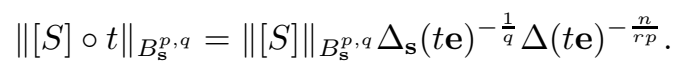

On the other hand, it is easier to determine the dual of a Besov space by working with its discrete version. Since the general case consists in a plain adaptation of the corresponding result $[\mathbf{2}, 3.27]$, we omit the proof of the next statement.

Proposition 3.14. Let $p, q$ belong to $] 1,+\infty\left[\right.$ and $\mathbf{s}$ to $\mathbb{R}^{r}$. Then, the dual space of $B_{\mathbf{S}}^{p, q}$ and $B_{-\left(q^{\prime}-1\right) \mathbf{s}}^{p^{\prime}, q^{\prime}}$ are isomorphic, with a duality pairing given by

$$
\underset{B_{-\left(q^{\prime}-1\right) \mathbf{s}}^{p^{\prime}, q^{\prime}}}{ }\langle[U],[S]\rangle_{B_{\mathbf{s}}^{p, q}} \doteq \sum_{j \in \mathbb{N}}\left\langle U * \widetilde{\chi}_{j},\left(S * \chi_{j}\right)^{\sim}\right\rangle
$$

which is independent of the representatives chosen for $[U],[S]$ and of the functions $\chi_{j}, \widetilde{\chi}_{j}$ (here, in addition to the requirements made on both families $\left(\widehat{\chi}_{j}\right)$ and $\left(\widehat{\widetilde{\chi}}_{j}\right)$ prior to Definition 3.10 , we are also assuming $\sum_{j} \widehat{\chi}_{j}=1_{\Omega}$ and $\left.\widehat{\widetilde{\chi}}_{j} \widehat{\chi}_{j}=\widehat{\chi}_{j}\right)$.

Note however that $(3.18)$ can also be given a continuous interpretation, for which it is sufficient to consider any pair of functions $\chi, \tilde{\chi} \in \mathcal{D}_{\Omega}$ such that $c(\chi)=1, c(\tilde{\chi}) \neq 0$ and $\widehat{\widetilde{\chi}} \hat{\chi}=\widehat{\chi}$. Indeed, simply let

$$
\underset{B_{-\left(q^{\prime}-1\right) \mathbf{s}}^{p^{\prime}, q^{\prime}}}{ }\langle[U],[S]\rangle_{B_{\mathbf{s}}^{p, q}} \doteq \int_{T^{*}}\left\langle U * \widetilde{\chi}_{\tau},\left(S * \chi_{\tau}\right)^{\varkappa}\right\rangle d \tau,
$$

and note that the two formulas agree (with $\left.(2 \pi)^{-n}\langle\widehat{U}, \widehat{f}\rangle\right)$ if $S \doteq f$ is in the dense subspace $\mathcal{D}_{\Omega}$, and that for both the estimate

$$
|\langle[U],[S]\rangle| \leq\|[U]\|_{B_{-\left(q^{\prime}-1\right) \mathbf{s}}^{p^{\prime}, q^{\prime}}}\|[S]\|_{B_{\mathbf{s}}^{p, q}}
$$

holds for arbitrary $[U],[S]$, by applying Hölder inequality twice.

\subsection{Multipliers.}

Definition 3.15. For $p$ in $[1,+\infty]$, we let $M_{p}$ denote the Banach space of $L^{p}(V, d x)$ multipliers with the induced norm as a subspace of the bounded linear operators from $L^{p}(V, d x)$ into itself.

$M_{p}$ being a non-separable space (it contains the uncountable, discrete subset formed by translation operators), weak measurability of an $M_{p}$-valued function is no longer sufficient for implementing a Bochner integral. Therefore, we need the following (standard) assumption, see e.g. $[19]$ : 
Definition 3.16. A $B$-valued function $f$ defined on a measure space $X$ is said strongly measurable if there exists a sequence $\left(s_{n}\right)_{n \in \mathbb{N}}$ of $B$-valued step-functions on $X$ such that

$$
s_{n}(x) \longrightarrow_{n} f(x)
$$

in the norm topology of $B$, for a.e. $x$ in $X$.

If $f$ is strongly measurable into a Banach space $B$, Pettis theorem in $[19$, V.4 $]$ shows that the map

$$
x \in X \longmapsto\|f(x)\|_{B}
$$

is measurable. If, in addition, $X$ is a separable topological space, then continuity of $f$ is enough to insure its strong measurability.

Now we are ready to state our main contribution to this section:

Theorem 3.17. Let $\tau \in T^{*} \stackrel{m}{\longmapsto} m_{\tau} \in M_{p}$ be a strongly measurable function, for some $p$ in $[1,+\infty]$, and assume that there exist $\mathbf{t} \in \mathbb{R}^{r}$ and $R_{0}>0$ such that

1) For every $\tau$ in $T^{*}$ the support of the multiplier $m_{\tau}$ is contained in $B\left(\tau \mathbf{e}, R_{0}\right)$.

2) For every positive $R$, the inequality

$$
\int_{B(\eta, R)}\left\|m_{\tau}\right\|_{M_{p}} d \tau \preceq_{R} \Delta_{\mathbf{t}}^{*}(\eta \mathbf{e})
$$

holds, independently of $\eta \in T^{*}$.

Then, the correspondence

$$
\xi \in \Omega \longmapsto \int_{T^{*}} m_{\tau}(\xi) d \tau
$$

well-defines an $L_{\text {loc. }}^{\infty}$ function $\widetilde{m}$, and the linear operator $T_{\widetilde{m}}$ mapping $f$ in $\mathcal{D}_{\Omega}$ to $\mathcal{F}^{-1}(\widetilde{m} \hat{f})$ in $\mathcal{S}_{\bar{\Omega}}^{\prime}$ uniquely extends to a bounded operator from $B_{\mathbf{s}}^{p, q}$ into $B_{\mathbf{s}+q \mathbf{t}^{*}}^{p, q}$, for any $q$ in $\left[1,+\infty\left[\right.\right.$ and $\mathbf{s}$ in $\mathbb{R}^{r}$.

We shall compute Besov norms w.r.t. a function $\psi$ in $\mathcal{D}_{\Omega}$ satisfying $c(\psi) \neq 0$; moreover, we fix $R_{1}>0$ such that the support of $\widehat{\psi}$ is contained in $B\left(\mathbf{e}, R_{1}\right)$.

Proof: Assumption (3.21) easily implies that the integral

$$
\int_{E} m_{\tau} d \tau
$$

exists in $M_{p}$, for every $d$-bounded measurable subset $E$ of $T^{*}$. In particular, since $M_{p}$ continuously embeds into $L^{\infty}(V)$ and $T^{*}$ is $\sigma$-compact, the 
first condition about the supports of the multipliers $m_{\tau}$ really means that $\widetilde{m}$ is an almost everywhere defined, measurable function in $L_{\text {loc. }}^{\infty}$. Therefore, $T_{\widetilde{m}}(f)$ belongs to $C_{0}$ for $f$ in $\mathcal{D}_{\Omega}$, and as a consequence the map

$$
\tau \in T^{*} \longmapsto T_{\widetilde{m}}(f) * \psi_{\tau}
$$

is continuous into $L^{p}(V, d x)$ (or into $C_{0}$, if $p=+\infty$ ), so that we only need the norm estimate

$$
\left\|T_{\widetilde{m}}(f)\right\|_{B_{\mathbf{s}+q^{*}}^{p, q}} \preceq\|f\|_{B_{\mathbf{s}}^{p, q}}
$$

for $f$ in $\mathcal{D}_{\Omega}$ to conclude, by the density result in Theorem 3.11. But for every $\eta$ in $T^{*}$ we have

$$
\left\|T_{\widetilde{m}}(f) * \psi_{\eta}\right\|_{p} \leq\left\|\widetilde{m} \widehat{\theta}_{\eta}\right\|_{M_{p}}\left\|f * \psi_{\eta}\right\|_{p}
$$

where $\widehat{\theta}$ is a Schwartz function which is identically 1 on the support of $\widehat{\psi}$ and whose support is contained in $B\left(\mathbf{e}, R_{1}\right)$. Thus, if we let

$$
\widetilde{m}_{\eta} \doteq \int_{B\left(\eta, R_{0}+R_{1}\right)} m_{\tau} d \tau
$$

we have $\widetilde{m} \widehat{\theta}_{\eta}=\widetilde{m}_{\eta} \widehat{\theta}_{\eta}$, so that by $(3.21)$ we obtain the estimate

$$
\begin{aligned}
\left\|\widetilde{m} \widehat{\theta}_{\eta}\right\|_{M_{p}} & \preceq\left\|\widetilde{m}_{\eta}\right\|_{M_{p}}\left\|\theta_{\eta}\right\|_{1} \\
& \preceq \int_{B\left(\eta, R_{0}+R_{1}\right)}\left\|m_{\tau}\right\|_{M_{p}} d \tau \preceq \Delta_{\mathbf{t}}^{*}(\eta \mathbf{e}) .
\end{aligned}
$$

Then, the desired inequality (3.22) trivially follows from (3.23) and (3.24).

Remark 3.18. It is even sufficient to assume that (3.21) holds for a single $\widetilde{R}$, since then we can extend its validity to arbitrary $R$ by the finite intersection property of a Whitney decomposition and Lemma 2.1.

At last, we take up the functional calculus for box operators on Besov spaces.

For $\left.u=\left(u_{1}, \ldots, u_{r}\right) \in\right] 0,+\infty\left[{ }^{r}\right.$ and $\mathbf{t} \in \mathbb{C}^{r}$, let $u^{\mathbf{t}} \doteq u_{1}^{t_{1}} \cdot \ldots \cdot u_{r}^{t_{r}}$.

Definition 3.19. Let $n \doteq \operatorname{dim} V$ and $M$ belong to $C^{n+1}(] 0,+\infty\left[^{r}\right)$.

We say that $M$ satisfies a t-shifted Marcinkiewicz-type condition for some $\mathbf{t}$ in $\mathbb{C}^{r}$ if $u^{-\mathbf{t}} M(u)$ is a Marcinkiewicz multiplier on $] 0,+\infty\left[^{r}\right.$, i.e., if

$$
\sup \left\{\left|u^{\alpha} \partial^{\alpha}\left(u^{-\mathbf{t}} M(u)\right)\right|:|\alpha| \leq n+1, u \in\right] 0,+\infty\left[^{r}\right\}<+\infty .
$$

Equivalently, we are requiring that

$$
\sup \left\{u^{\alpha-\Re e \mathbf{t}}\left|\partial^{\alpha} M(u)\right|:|\alpha| \leq n+1, u \in\right] 0,+\infty\left[^{r}\right\}<+\infty,
$$

as it can be easily shown by induction on the length of $\alpha$. 
Corollary 3.20. Let $M$ satisfy a $\mathbf{t}$-shifted Marcinkiewicz-type condition for some $\mathbf{t}$ in $\mathbb{C}^{r}$, and define

$$
\widetilde{m} \doteq M \circ\left(\Delta_{1}^{*}, \frac{\Delta_{2}^{*}}{\Delta_{1}^{*}}, \ldots, \frac{\Delta}{\Delta_{r-1}^{*}}\right) .
$$

Then, the linear operator $T_{M}$ initially defined on $\mathcal{D}_{\Omega}$ by

$$
T_{M} f \doteq \frac{1}{(2 \pi)^{n}} \mathcal{F}^{-1}(\widetilde{m} \widehat{f})
$$

extends to a bounded, linear operator from $B_{\mathbf{s}}^{p, q}$ into $B_{\mathbf{s}+q \Re e \mathbf{t}^{*}}^{p, q}$, for every $p$ in $] 1,+\infty\left[, q\right.$ in $\left[1,+\infty\left[\right.\right.$ and $\mathbf{s}$ in $\mathbb{R}^{r}$.

Note that if $M$ is $C^{\infty}$, then $T_{M}$ maps $\mathcal{D}_{\Omega}$ into itself.

Proof: We fix a function $\psi$ in $\mathcal{D}_{\Omega}$ satisfying (3.2). Then, the claim is that the estimate

$$
\left\|\widehat{\psi}_{\tau} \widetilde{m}\right\|_{M_{p}} \preceq \Delta_{\Re e \mathbf{t}}^{*}(\tau \mathbf{e})
$$

holds, with constants independent of $\tau$ in $T^{*}$. Once this is done, we have that the $M_{p}$-valued function

$$
\tau \in T^{*} \longmapsto m_{\tau} \doteq \widehat{\psi}_{\tau} \widetilde{m}
$$

is continuous, so that we can directly conclude on behalf of Theorem 3.17, since $\int_{T^{*}} \widehat{\psi}_{\tau}(\xi) d \tau=1$ for $\xi$ in $\Omega$. But

$$
\begin{aligned}
\left\|m_{\tau}\right\|_{M_{p}} & \preceq\left\|\mathcal{F}^{-1} m_{\tau}\right\|_{L^{1}(V, d x)} \\
& \preceq p_{n+1}\left(\widehat{\psi} M\left(\Delta_{1}^{*}(\tau \mathbf{e}) \Delta_{1}^{*}, \ldots, \frac{\Delta(\tau \mathbf{e}) \Delta}{\Delta_{r-1}^{*}(\tau \mathbf{e}) \Delta_{r-1}^{*}}\right)\right),
\end{aligned}
$$

and letting $u_{\tau}$ denote the function

$$
\left.\xi \in \Omega \longmapsto\left(\Delta_{1}^{*}(\tau \xi), \ldots, \frac{\Delta(\tau \xi)}{\Delta_{r-1}^{*}(\tau \xi)}\right) \in\right] 0,+\infty\left[^{r}\right.
$$

we can prove by induction that for $|\alpha| \leq n+1$ there exist (regular) functions $h_{\beta}$ on $\Omega$, which may depend on $\alpha$ but not on $\tau$, such that

$$
\partial^{\alpha}\left(M \circ u_{\tau}\right)=\sum_{|\beta| \leq|\alpha|} \Delta_{\beta}^{*}(\tau \mathbf{e})\left(\left(\partial^{\beta} M\right) \circ u_{\tau}\right) h_{\beta} .
$$

The support of $\widehat{\psi}$ being compact, we obtain (3.26) from (3.25), (3.27) and Lemma 2.1. 
Remark 3.21. Therefore, for $\mathbf{t}$ in $\mathbb{C}^{r}$ we can define the operator

$$
\square^{\mathbf{t}}: B_{\mathbf{s}}^{p, q} \longrightarrow B_{\mathbf{s}+q \Re e \mathbf{t}^{*}}^{p, q}
$$

as $T_{M^{\mathrm{t}}}$, that is, by applying Corollary 3.20 to the function

$$
u \in] 0,+\infty\left[{ }^{r} \longmapsto M^{\mathbf{t}}(u) \doteq u^{\mathrm{t}} .\right.
$$

In this case, more can be said: indeed, any $\square^{\mathrm{t}}$ sets up an isomorphism of Banach spaces, for $p, q$ and $\mathbf{s}$ as in the corollary, its inverse being $\square^{-\mathbf{t}}$.

In particular, if $\mathbf{t}$ belongs to $\mathbb{N}^{r}$ and $t_{1} \geq t_{2} \geq \cdots \geq t_{r}$, the operator $\square^{\mathbf{t}}$ is $(2 \pi)^{-n}$ times the generalized wave operator on $\Omega$ (whence the $\square$ notation), which is the differential operator $\Delta_{\mathbf{t}}^{*}\left[\frac{1}{i} \partial_{x}\right]$ of degree $t_{1}+\cdots+t_{r}$ characterized for $\xi \in V$ by the equality

$$
\Delta_{\mathbf{t}}^{*}\left[\frac{1}{i} \partial_{x}\right] e^{i(x \mid \xi)}=\Delta_{\mathbf{t}}^{*}(\xi) e^{i(x \mid \xi)} .
$$

\section{Boundary values of $\mathcal{A}_{\mathrm{s}}^{p, q}$ functions}

\subsection{The Cauchy extension operator.}

Motivated by (3.17) and Theorem 2.7, we now address the question whether or not we can represent a Bergman space $\mathcal{A}_{\mathrm{s}}^{p, q}$ through suitable boundary values in the corresponding Besov space $B_{\mathbf{s}}^{p, q}$. Following [2], this is done by studying the Cauchy extension operator $\mathcal{E}$, which constitutes the bridge between the two families of spaces.

$\mathcal{E}$ is initially defined on $\mathcal{D}_{\Omega}$ by composing the Fourier and the Laplace transform (modulo multiplicative constants), mapping $f$ to the function

$$
z \in T_{\Omega} \longmapsto(\mathcal{E} f)(z) \doteq \frac{1}{(2 \pi)^{n}}(\mathcal{L} \widehat{f})(z)=\frac{1}{(2 \pi)^{n}} \int_{\Omega} e^{i(z \mid \xi)} \widehat{f}(\xi) d \xi .
$$

The next proposition allows to extend the definition of $\mathcal{E}$ to all of $B_{\mathbf{s}}^{p, q}$. Note however that the said extension does not necessarily coincide with the classical Fourier-Laplace transform $\mathcal{L} \circ \mathcal{F}$, see Remark 4.3. This notwithstanding, we shall continue to denote by $\mathcal{E}$ the operator which we are going to produce on $B_{\mathbf{s}}^{p, q}$.

Proposition 4.1 is in $[\mathbf{2}, 3.43]$ for the case $\mathbf{s}=(\nu, \ldots, \nu)$, but for the sake of clarity we give the proof here, using the continuous description of $B_{\mathbf{s}}^{p, q}$ and Theorem 3.17 . 
Proposition 4.1. Let $p, q$ belong to $] 1,+\infty\left[\right.$ and $\mathbf{s}$ to $\mathbb{R}^{r}$. In addition, assume that

$$
q\left(\mathrm{~g}_{0}-\left(\frac{n}{r p}, \ldots, \frac{n}{r p}\right)\right)<\mathbf{s}^{*}+\mathrm{g}_{0} .
$$

Then, $\mathcal{E}$ is continuous from $\left(\mathcal{D}_{\Omega},\|\|_{B_{\mathrm{s}}^{p, q}}\right)$ into $\mathcal{H}$ ol $\left(T_{\Omega}\right)$, endowed with the Fréchet topology of uniform convergence over compact subsets of $T_{\Omega}$, and it can therefore be extended to a bounded linear operator on all of $B_{\mathbf{s}}^{p, q}$. Moreover, the equivalence class of the distribution $(\mathcal{E}[S])_{y}$ belongs to $B_{\mathbf{s}}^{p, q}$ for every $[S]$ in $B_{\mathbf{s}}^{p, q}$ and $y$ in $\Omega$, and the net so obtained converges to $[S]$ in norm as $y$ approaches 0 inside $\Omega$, i.e.

$$
\lim _{\Omega \ni y \rightarrow 0}\left\|\left[(\mathcal{E}[S])_{y}\right]-[S]\right\|_{B_{\mathbf{s}}^{p, q}=0 .}
$$

Proof: First of all, we prove that there exists a constant $C>0$ such that the estimate

$$
|(\mathcal{E} f)(z)| \leq C \Delta_{\mathbf{s}}(\Im m z)^{-\frac{1}{q}} \Delta(\Im m z)^{-\frac{n}{r p}}\|f\|_{B_{\mathbf{s}}^{p, q}}
$$

holds, for every $z$ in $T_{\Omega}$ and $f$ in $\mathcal{D}_{\Omega}$. This is sufficient to deduce that $\mathcal{E}$ is continuous from $\mathcal{D}_{\Omega}$ into $\operatorname{Hol}\left(T_{\Omega}\right)$. Now, by invariance it is even enough to show (4.2) with $z=i \mathbf{e}$. But we can interpret $(\mathcal{E} f)(i \mathbf{e})$ as the duality pairing between $\mathcal{F}^{-1}\left(1_{\Omega} e^{-(\mathbf{e} \mid \cdot)}\right)$ and $f$, if the former belongs to $B_{-\left(q^{\prime}-1\right) \mathbf{s}}^{p^{\prime}, q^{\prime}}$. Still, if $g$ is any $C^{\infty}(V)$ function which is identically 1 on $\Omega$, we have that

$$
\left\|\mathcal{F}^{-1}\left(1_{\Omega} e^{-(\mathbf{e} \mid \cdot)}\right)\right\|_{B_{-\left(q^{\prime}-1\right) \mathbf{s}}^{p^{\prime}, q^{\prime}}}=\left\|\left(\tau \longmapsto \mathcal{F}^{-1}\left(g e^{-(\mathbf{e} \mid \cdot)}\right) * \psi_{\tau}\right)\right\|_{L_{-\left(q^{\prime}-1\right) \mathbf{s}}^{p^{\prime}, q^{\prime}}}\left(T^{*}\right) .
$$

If we also choose a $g$ which has bounded derivatives of all orders and is supported in the sum of $\Omega$ and a euclidean ball centered at the origin, then $g e^{-(\mathbf{e} \cdot \cdot)}$ is a Schwartz function on $V$, so that we can conclude the proof of (4.2) on behalf of duality and the following lemma, which partly explains the restrictive condition in (4.1):

Lemma 4.2. Let $(p, q) \in[1,+\infty]^{2} \backslash\{(1,+\infty)\}, \mathbf{s} \in \mathbb{R}^{r}$ and assume that

$$
q\left(\frac{n}{r p^{\prime}}, \ldots, \frac{n}{r p^{\prime}}\right)>\mathbf{s}^{*}+\mathbf{g}_{\mathbf{0}} .
$$

Then, the map

$$
f \in \mathcal{S}(V) \longmapsto\left(\tau \longmapsto f * \psi_{\tau}\right) \in L_{\mathbf{s}}^{p, q}\left(T^{*}\right)
$$

is continuous. 
Proof: For a general Schwartz function $f$ we still have that for $m=0$ (3.4) holds; as a consequence, (3.3) holds too, with $\mathbf{t}=0$. Thus, if $q=+\infty$ the norm estimate is trivial, and in this case $p \neq 1$ is used to guarantee that the element $\left(\tau \longmapsto f * \psi_{\tau}\right)$ also belongs to $C_{0}\left(L^{p}\right)$, owing to the presence of the factor $\Delta(\tau \mathbf{e})^{\frac{n}{r p^{\prime}}}$. For a finite $q$ the result follows by (3.5) and (2.12).

We continue with the proof of the proposition: note that for $f$ in $\mathcal{D}_{\Omega}$ and $y$ in $\Omega$ we have that

$$
(\mathcal{E} f)_{y}=\frac{1}{(2 \pi)^{n}} \mathcal{F}^{-1}\left(e^{-(y \mid \cdot)} \widehat{f}\right) ;
$$

in particular, $(\mathcal{E} f)_{y}$ belongs to $\mathcal{D}_{\Omega}$ for every $y$ in $\Omega$, and it converges to $f$ in the Schwartz topology, as $y$ tends to 0 inside $\Omega$. By Lemma 3.5 we have convergence in $B_{\mathbf{s}}^{p, q}$. Finally, we claim that $\left[(\mathcal{E}[S])_{y}\right]$ belongs to $B_{\mathbf{s}}^{p, q}$ for arbitrary $[S] \in B_{\mathbf{s}}^{p, q}$ and $y \in \Omega$, and that it satisfies

$$
\left\|\left[(\mathcal{E}[S])_{y}\right]\right\|_{B_{\mathbf{S}}^{p, q}} \preceq\|[S]\|_{B_{\mathbf{S}}^{p, q}}
$$

independently of $[S]$ and $y$, which would allows us to prove that $\left[(\mathcal{E}[S])_{y}\right]$ tends to $[S]$ in the norm of $B_{\mathbf{s}}^{p, q}$ as $y$ goes to 0 inside $\Omega$ by a standard $3 \epsilon$ argument.

But $\widetilde{m}_{y} \doteq \frac{1_{\Omega}}{(2 \pi)^{n}} e^{-(y \mid \cdot)}$ is a uniformly bounded family of $B_{\mathbf{s}}^{p, q}$ multipliers for $y$ in $\Omega$, by Theorem 3.17. Therefore, (4.4) holds for the corresponding operators $T_{y} \doteq T_{\widetilde{m}_{y}}$, and we are only left to show that

$$
\psi_{\tau} * T_{y}[S]=\psi_{\tau} *(\mathcal{E}[S])_{y}
$$

for every $\tau \in T^{*}$. In turn, since the estimates (4.2) imply that the operator $[S] \in B_{\mathbf{s}}^{p, q} \mapsto \psi_{\tau} *(\mathcal{E}[S])_{y} \in C_{0}(V)$ is bounded, (4.5) finally follows by a density argument.

Remark 4.3. Due to the presence of equivalence classes, $\mathcal{E}$ does not agree with the usual Fourier-Laplace transform $[\mathbf{1 3}, \S 7.4]$ of distributions supported in $\bar{\Omega}$ : for instance, $\mathcal{E}\left(\mathcal{S}_{\partial \Omega}^{\prime}\right)=0$, while $\mathcal{L} \circ \mathcal{F}$ is injective. However, note that, apart from a scalar factor, $\mathcal{E}[S]$ does coincide with $\mathcal{L} \widehat{S}$ whenever $\widehat{S}$ is compactly supported in $\Omega$ (and of course, if $\|[S]\|_{B_{\mathbf{s}}^{p, q}}$ is finite).

We will be able to say more about Remark 4.3 after the next lemma, which allows us to identify $B_{\mathbf{s}}^{p, q}$ with a subspace of $\mathcal{S}^{\prime}(V)$ by choosing a natural representative in each equivalence class. The formulation of the result is in $[\mathbf{2}, 3.38]$ for the discrete setting, but for future use we prefer to give a proof adapted to the continuous description of $B_{\mathbf{s}}^{p, q}$. 
Lemma 4.4. Assume that $p, q$ belong to $] 1,+\infty\left[\right.$, $\mathbf{s}$ is in $\mathbb{R}^{r}$ and that condition (4.1) holds. Then, for every $[S]$ in $B_{\mathbf{s}}^{p, q}$, the integral

$$
\int_{T^{*}} S * \psi_{\tau} d \tau
$$

distributionally defines an element $S^{\sharp}$ in the equivalence class of $[S]$. Moreover, the discrete decomposition

$$
S^{\sharp}=\sum_{j} S * \chi_{j}
$$

holds and the limit

$$
\lim _{\Omega \ni y \rightarrow 0}(\mathcal{E}[S])_{y}=S^{\sharp}
$$

exists, both with convergence in $\mathcal{S}^{\prime}(V)$. In particular, $S^{\sharp}$ is independent of the representative chosen for $[S]$ and $\mathcal{E}$ is injective on $B_{\mathbf{s}}^{p, q}$.

Note that (4.6) and (4.7) do not depend on the choice of $\psi$ or $\chi_{j}$ 's provided they satisfy $c(\psi)=1$ and $\sum_{j} \widehat{\chi}_{j}=1_{\Omega}$.

Proof: Formula (4.6) simply means that for $g$ in $\mathcal{S}(V)$ we let

$$
\left\langle S^{\sharp}, g\right\rangle \doteq \int_{T^{*}}\left\langle S * \psi_{\tau}, g\right\rangle d \tau .
$$

Now, if $\theta \in \mathcal{D}_{\Omega}$ is such that $c(\theta) \neq 0$ and $\widehat{\theta} \widehat{\psi}=\widehat{\psi}$, by applying Hölder inequality twice as in (3.20) we obtain

$$
\begin{aligned}
\left|\left\langle S^{\sharp}, g\right\rangle\right| & \leq \int_{T^{*}}\left|\left\langle S * \psi_{\tau},\left(\check{g} * \theta_{\tau}\right)^{\sim}\right\rangle\right| d \tau \\
& \preceq\|[S]\|_{B_{\mathbf{s}}^{p, q}}\left\|\left(\tau \longmapsto \check{g} * \theta_{\tau}\right)\right\|_{\left(L_{\mathbf{s}}^{p, q}\left(T^{*}\right)\right)^{*}},
\end{aligned}
$$

and the right-hand side is a finite quantity for any $g$ in $\mathcal{S}(V)$ because of conditions (4.1) and Lemma 4.2. This is sufficient to conclude that $S^{\sharp}$ is in $\mathcal{S}^{\prime}(V)$, and then easy manipulations with (4.6) show that it also belongs to $\mathcal{S} \frac{\prime}{\Omega}$. Moreover, if $h$ is a Schwartz function supported in $\Omega$, we can apply Proposition 3.2 to $\widehat{h}$ obtaining that

$$
\left\langle\widehat{S^{\sharp}}, h\right\rangle=\int_{T^{*}}\left\langle S * \psi_{\tau}, \widehat{h}\right\rangle d \tau=\left\langle\check{S}, \int_{T^{*}} \widehat{\breve{h}} * \psi_{\tau} d \tau\right\rangle=\langle\widehat{S}, h\rangle,
$$

or equivalently $\left[S^{\sharp}\right]=[S]$. Next, note that the estimate

$$
\left|\left\langle\sum_{j} S * \chi_{j}, g\right\rangle\right| \preceq\|[S]\|_{B_{s}^{p, q}\left\|\left(\check{g} * \chi_{j}\right)_{j}\right\|_{\left(\ell_{s}^{p, q}\right)^{*}}}
$$


holds as well for every $g \in \mathcal{S}(V)$, by a trivial adaptation of the proofs of (4.9) and Lemma 4.2 to the discrete setting. Therefore, the map

$$
[S] \in B_{\mathbf{s}}^{p, q} \longmapsto S^{\sharp}-\sum_{j} S * \chi_{j} \in \mathcal{S}^{\prime}(V)
$$

is continuous, and it clearly vanishes on $\mathcal{D}_{\Omega}$, so that (4.7) is established by density. Finally, the last part of the statement follows from Proposition 4.1 once we note that

$$
\left[(\mathcal{E}[S])_{y}\right]^{\sharp}=(\mathcal{E}[S])_{y}
$$

in $\mathcal{S}^{\prime}(V)$, for every $[S] \in B_{\mathbf{s}}^{p, q}$ and $y \in \Omega$. But $(\mathcal{E}[S])_{y}=\sum_{j \in \mathbb{N}}\left(\mathcal{E}\left[S * \chi_{j}\right]\right)_{y}$ in $C_{0}(V)$ and therefore in $\mathcal{S}^{\prime}(V)$, by (3.13), (4.2) and the continuity of $\mathcal{E}$. Hence, it is enough to show

$$
\left(\mathcal{E}\left[S * \chi_{j}\right]\right)_{y}=(\mathcal{E}[S])_{y} * \chi_{j}
$$

for every $j \in \mathbb{N}$, and in turn this last equality is a consequence of (4.5).

Remark 4.5. Note that as a by-product of (4.7) and Remark 4.3, under the assumptions made in (4.1) one can also extend the validity of

$$
\mathcal{E}[S]=(2 \pi)^{-n} \mathcal{L} \widehat{S^{\sharp}}
$$

to every $[S]$ in $B_{\mathbf{s}}^{p, q}$, for $\mathcal{L}$ is continuous on $\mathcal{S}^{\prime}(V)$, see [13, VII.4.2].

From now on, condition (4.1) and its dual (4.3) will appear in an increasing number of situations, each time assuming the role of critical indices for some property. This is indeed the case in Proposition 4.1 and in Lemma 4.2, which do not hold beyond these indices (at least for $p$ and $q$ strictly between 1 and $+\infty$ ). The proof of these two observations is a simple modification of the one presented in [2, 3.48], and so we skip it.

\subsection{The proof of Theorem 1.2 .}

We now prove a partial result in the direction of representing a Bergman space by means of the Cauchy operator defined on the corresponding Besov space, extending $[\mathbf{2}, 1.7]$. The proof is slightly simpler though, for the continuous notation is employed.

Theorem 4.6. Let $p$ and $q$ belong to $] 1,+\infty\left[, \mathbf{s}\right.$ to $\mathbb{R}^{r}$ and assume that condition (4.1) holds. Then, $\mathcal{E}\left(B_{\mathbf{s}}^{p, q}\right)$ contains $\mathcal{A}_{\mathbf{s}}^{p, q}$ and the operator $\mathcal{E}^{-1}$ defined from $\mathcal{A}_{\mathbf{s}}^{p, q}$ into $B_{\mathbf{s}}^{p, q}$ is bounded with dense image. In particular,

$$
\lim _{\Omega \ni y \rightarrow 0}\left\|\left[F_{y}\right]-\mathcal{E}^{-1} F\right\|_{B_{\mathbf{s}}^{p, q}}=0
$$

for every $F$ in $\mathcal{A}_{\mathbf{s}}^{p, q}$. 
Proof: By Theorem 2.15 we may also assume that $\mathbf{s}>\mathbf{g}_{\mathbf{0}}$, for else there is nothing to prove. In this case, we know from Proposition 2.14 that $\mathcal{A}_{\mathbf{s}}^{p, q} \cap \mathcal{H}^{2}$ is a dense subspace of $\mathcal{A}_{\mathbf{s}}^{p, q}$. Therefore, to any $F \in \mathcal{A}_{\mathbf{s}}^{p, q} \cap \mathcal{H}^{2}$ we can associate $\widehat{g}$ in $L^{2}(\Omega, d \xi)$ such that $F=(2 \pi)^{-n} \mathcal{L} \widehat{g}$, by Theorem 2.7. Moreover, $g=\sum_{j \in \mathbb{N}} g * \chi_{j}$ in $L^{2}(V, d x)$ (and consequently in $\mathcal{S}^{\prime}(V)$ ) by Plancherel formula and dominated convergence. Thus, if we can show that $g$ is in $B_{\mathbf{S}}^{p, q}$, we also find that $F=\mathcal{E}[g]$, by (4.12). But

$$
\begin{aligned}
\left\|g * \psi_{\tau}\right\|_{p} & \preceq\left\|\mathcal{F}^{-1}\left(\widehat{g} e^{-\left(\tau^{*^{-1}} \mathbf{e} \mid \cdot\right)}\right)\right\|_{p}\left\|\mathcal{F}^{1}\left(\widehat{\psi} e^{(\mathbf{e} \mid \cdot)}\right)\right\|_{1} \\
& =\left\|F_{(\tau \mathbf{e})^{-1}}\right\|_{p}\left\|\mathcal{F}^{-1}\left(\widehat{\psi} e^{(\mathbf{e} \mid \cdot)}\right)\right\|_{1} .
\end{aligned}
$$

Therefore,

$$
\begin{aligned}
\|[g]\|_{B_{\mathbf{s}}^{p, q}}^{q} & \preceq \int_{T^{*}}\left\|F_{(\tau \mathbf{e})^{-1}}\right\|_{p}^{q} \Delta_{\mathbf{S}}\left((\tau \mathbf{e})^{-1}\right) d \tau \\
& =\int_{\Omega}\left\|F_{\xi^{-1}}\right\|_{p}^{q} \Delta_{\mathbf{S}}\left(\xi^{-1}\right) \frac{d \xi}{\Delta(\xi)^{\frac{n}{r}}}=\|F\|_{\mathcal{A}_{\mathbf{s}}^{p, q}}^{q},
\end{aligned}
$$

for the invariant measure (2.2) is preserved under inversion, see [7, II.3.3]. In the general case we may proceed by density. Finally, we show that the image of $\mathcal{E}^{-1}$ contains $\mathcal{D}_{\Omega}$. So, pick up $g$ in $\mathcal{D}_{\Omega}$ and let $H$ denote the support of $\widehat{g}$ and $\widehat{\vartheta}$ be any function in $C_{c}^{\infty}(\Omega)$ which is identically 1 on $H$. Then, there exists $\gamma_{H}>0$ such that

$$
\left\|(\mathcal{E} g)_{y}\right\|_{p} \preceq\left\|g * \mathcal{F}^{-1}\left(e^{-(y \mid \cdot)} \widehat{\vartheta}\right)\right\|_{p} \preceq_{H}\|g\|_{p} e^{-\gamma_{H}(y \mid \mathbf{e})}
$$

independently of $y$ in $\Omega$. Since $\mathbf{s}>\mathbf{g}_{\mathbf{0}}$, we can easily conclude by (2.3) that $\|\mathcal{E} g\|_{\mathcal{A}_{\mathbf{s}}^{p, q}}$ is finite.

In fact, Lemma 4.4 is more precise than Theorem 4.6 as to (4.13), since in addition it asserts that the limit of $F_{y}$ exists in $\mathcal{S}^{\prime}(V)$ as well, for $y$ tending to 0 inside $\Omega$ and $F$ in $\mathcal{A}_{\mathbf{s}}^{p, q}$. What we can still do in the hypothesis of Theorem 4.6 is to explicitly determine this limit, that is, $\left(\mathcal{E}^{-1} F\right)^{\sharp}$.

This part is new if compared to $[\mathbf{2}]$, and it gives the "correct" definition for the operator $\mathcal{E}^{-1}$, according to Lemma 4.4 .

Heuristically, the idea comes from "discretizing" (4.12) with $\widehat{S}=\widehat{g}$ in $L^{2}(\Omega, d \xi)$ at height $y$ and then let $y$ tend to 0 :

$$
F_{y}=\sum_{j \in \mathbb{N}} \int_{B\left(\xi_{j}, 2\right)} \widehat{g}(\xi) \widehat{\chi}_{j}(\xi) e^{i(\cdot+i y \mid \xi)} d \xi \sim \sum_{j \in \mathbb{N}} e^{-\left(y \mid \xi_{j}\right)}\left(F_{\xi_{j}^{-1}} * \chi_{j}\right) .
$$


However, in order to make this argument work, we must introduce an appropriate invariance property which is most conveniently exploited in the continuous notation. In detail, once a function $\psi$ in $\mathcal{D}_{\Omega} \backslash\{0\}$ whose Fourier transform is everywhere non-negative on $\Omega$ has been fixed, define

$$
a(\psi) \doteq \int_{\Omega} e^{-(\xi \mid \mathbf{e})} \widehat{\psi}(\xi)\left(\Delta_{1}^{*}(\xi) \cdots \Delta_{r-1}^{*}(\xi)\right)^{-d} \frac{d \xi}{\Delta(\xi)},
$$

which is a strictly positive number, and for $F$ in $\mathcal{A}_{\mathbf{s}}^{p, q}$ let

$$
\left\langle F_{0}, h\right\rangle=a(\psi)^{-1} \int_{T^{*}}\left\langle F_{\tau^{*}-1} \mathbf{e}^{*} \psi_{\tau}, h\right\rangle d \tau \quad(h \in \mathcal{S}(V)) .
$$

Then, we claim that $F_{0}$ is a well defined distribution in $\mathcal{S}_{\bar{\Omega}}^{\prime}$ and the canonic representative of $\mathcal{E}^{-1} F$ in $B_{\mathbf{s}}^{p, q}$ at the same time.

First of all, proceeding as in (4.9) we obtain the inequality

$$
\left|\left\langle F_{0}, h\right\rangle\right| \preceq\left(\int_{\Omega}\left\|F_{\xi^{-1}}\right\|_{p}^{q} \Delta_{\mathbf{s}}\left(\xi^{-1}\right) \frac{d \xi}{\Delta(\xi)^{\frac{n}{r}}}\right)^{\frac{1}{q}}\left\|\left(\tau \longmapsto \check{h} * \psi_{\tau}\right)\right\|_{\left(L_{\mathbf{s}}^{p, q}\left(T^{*}\right)\right)^{*}}
$$

and therefore (4.14) and Lemma 4.2 show that $F_{0}$ is a tempered distribution whose Fourier transform is supported in $\bar{\Omega}$.

Then, note that there exists $R>0$ such that for every $\eta \in T^{*}$ we have

$$
\left\|F_{0} * \psi_{\eta}\right\|_{p}^{q} \preceq \int_{B(\eta, R)}\left\|F_{\tau^{*-1}}\right\|_{p}^{q} d \tau .
$$

In particular, by Lemma 2.1 and invariance we may estimate

$$
\begin{aligned}
\left\|\left[F_{0}\right]\right\|_{B_{\mathbf{s}}^{p, q}}^{q} & \preceq \int_{T^{*}}\left(\int_{B(\eta, R)}\left\|F_{\tau^{*-1}}\right\|_{p}^{q} d \tau\right) \Delta_{\mathbf{s}}\left((\eta \mathbf{e})^{-1}\right) d \eta \\
& \preceq \int_{T^{*}}\left(\int_{B(\tau, R)}\left\|F_{\tau^{*}-1}\right\|_{p}^{q} d \eta\right) \Delta_{\mathbf{s}}\left((\tau \mathbf{e})^{-1}\right) d \tau \\
& \preceq \int_{\Omega}\left\|F_{\xi^{-1}}\right\|_{p}^{q} \Delta_{\mathbf{s}}\left(\xi^{-1}\right) \frac{d \xi}{\Delta(\xi)^{\frac{n}{r}}}
\end{aligned}
$$

which finally is $\|F\|_{\mathcal{A}_{\mathbf{s}}^{p, q}}^{q}$, by (4.14) once more.

The next step consists in proving that $\left[F_{0}\right]^{\sharp}=F_{0}$, or equivalently, that $\left\langle F_{0}-\sum_{j=0}^{N} F_{0} * \chi_{j}, h\right\rangle$ tends to 0 as $N$ diverges, for every $h$ in $\mathcal{S}(V)$. But

$$
\left\langle F_{0}-\sum_{j=0}^{N} F_{0} * \chi_{j}, h\right\rangle=\left\langle F_{0}, h-\sum_{j=0}^{N} h * \check{\chi}_{j}\right\rangle,
$$


so that

$$
\left|\left\langle F_{0}, h-\sum_{j=0}^{N} h * \check{\chi}_{j}\right\rangle\right| \preceq\|F\|_{\mathcal{A}_{\mathbf{s}}^{p, q}}\left\|\left[\mathcal{F}^{-1}\left(1_{\Omega}\left(\widehat{\widehat{h}}-\sum_{j=0}^{N} \widehat{\bar{h} * \chi_{j}}\right)\right)\right]\right\|_{\left(B_{\mathbf{s}}^{p, q}\right)^{*}}
$$

by the estimate (4.16), and actually

$$
\left[\mathcal{F}^{-1}\left(1_{\Omega} \sum_{j=0}^{N} \widehat{\bar{h} * \chi_{j}}\right)\right]=\sum_{j=0}^{N}\left[\check{h} * \chi_{j}\right]
$$

tends to $\left[\mathcal{F}^{-1}\left(1_{\Omega} \widehat{\hat{h}}\right)\right]$ in $\left(B_{\mathbf{S}}^{p, q}\right)^{*}$ by (3.13) and Proposition 3.14, for

$$
\left\|\left[\mathcal{F}^{-1}\left(1_{\Omega} \widehat{\hat{h}}\right)\right]\right\|_{\left(B_{\mathbf{s}}^{p, q}\right)^{*}}=\left\|\left(\check{h} * \psi_{\tau}\right)_{\tau}\right\|_{\left(L_{\mathbf{s}}^{p, q}\left(T^{*}\right)\right)^{*}}
$$

is finite by Lemma 4.2 .

Finally, we show that $F_{0}$ is the limit of the net $\left(F_{y}\right)_{y}$ in $\mathcal{S}^{\prime}(V)$ as $y$ approaches 0 inside $\Omega$. By Theorem 4.6 and (4.17) we may assume that $F=\mathcal{E} g$ for some $\widehat{g}$ in $L^{2}(\Omega, d \xi)$. For $h$ in $\mathcal{S}(V)$ we then have

(4.18) $\left.a(\psi)\left\langle\widehat{F_{0}}, h\right\rangle=\int_{T^{*}}\left\langle\widehat{F_{\tau^{*}-1} \mathbf{e}}, h \widehat{\psi}_{\tau}\right\rangle d \tau=\int_{T^{*}}\left\langle e^{-\left(\tau^{*}-1\right.} \mathbf{e} \cdot \cdot\right) \widehat{g}, h \widehat{\psi}_{\tau}\right\rangle d \tau$.

But

$$
\int_{T^{*}} e^{-\left(\mathbf{e} \mid \tau^{-1} v\right)} \widehat{\psi}\left(\tau^{-1} v\right) d \tau=a(\psi)
$$

independently of $v$ in $\Omega$, by left invariance of $d \tau$ and (3.8), so that applying Fubini's theorem in (4.18) we can conclude that $F_{0}=g$, as claimed.

We record this result in a statement for future reference:

Corollary 4.7. Let $p$ and $q$ belong to $] 1,+\infty\left[, \mathbf{s}>\mathbf{g}_{0}\right.$ and assume that condition (4.1) holds. Then, the map

$$
F \in \mathcal{A}_{\mathbf{s}}^{p, q} \longmapsto F_{0} \in B_{\mathbf{s}}^{p, q}
$$

defines a bounded linear operator with a dense image, and $F=\mathcal{E}\left[F_{0}\right]$. Moreover, $F_{0}=\left[F_{0}\right]^{\sharp}$ is the limit of $F_{y}$ when $y$ tends to 0 inside $\Omega$, both in the norm of $B_{\mathbf{s}}^{p, q}$ and in $\mathcal{S}^{\prime}(V)$.

\subsection{The isomorphism between $\mathcal{A}_{\mathrm{s}}^{p, q}$ and $B_{\mathrm{s}}^{p, q}$.}

Corollary 4.7 embodies the fact that a function $F$ in $\mathcal{A}_{\mathrm{s}}^{p, q}$ has a boundary value $F_{0}$ in $B_{\mathbf{s}}^{p, q}$, and that we can get $F$ back extending $F_{0}$ to the tube domain over $\Omega$ by means of the Cauchy operator $\mathcal{E}$.

However, it still does not say if every distribution in $B_{\mathbf{s}}^{p, q}$ is obtained this way, that is, whether $\mathcal{E}\left(B_{\mathbf{s}}^{p, q}\right)$ properly contains $\mathcal{A}_{\mathbf{s}}^{p, q}$ or else, if these 
two spaces coincide. And in fact, we will see that both possibilities can occur, as $[\mathbf{2}, 1.9]$ already shows in case $\mathbf{s}=(\nu \ldots, \nu)$.

Therefore, we shall prove in a moment a strengthening of Theorem 4.6 stating that $\mathcal{E}\left(B_{\mathbf{s}}^{p, q}\right)=\mathcal{A}_{\mathbf{s}}^{p, q}$, though under some further restrictions on $p, q$ and $\mathbf{s}$, and afterwards we shall look for counterexamples. Even if some of this material clearly is an adaptation of the corresponding results in $[\mathbf{2}]$, we will give the proofs, to illustrate where the additional restrictions related to the weights $\Delta_{\mathrm{s}}$ come from.

Before doing this we restate condition (4.1): under the assumption $\mathbf{s}>$ $\mathrm{g}_{0}$ it is equivalent with

$$
q<Q_{\mathbf{s}}(p) \doteq \min _{j=1, \ldots, r} \frac{s_{j}+\frac{d}{2}(r-j)}{\left(\frac{d}{2}(r-j)-\frac{n}{r p}\right)_{+}},
$$

where for strictly positive $c$ and arbitrary (real) $a$ we let

$$
a_{+} \doteq \max \{a, 0\} \quad \text { and } \quad \frac{c}{0} \doteq+\infty .
$$

Note that the inequality $Q_{\mathbf{s}}(p)>p^{\prime}$ always holds, since $\mathbf{s}>\mathbf{g}_{\mathbf{0}}$; nevertheless, and unlike the situation in $[\mathbf{2}]$, here $Q_{\mathbf{s}}(p)<2$ can occur. At the other extreme, $Q_{\mathbf{s}}(p)=+\infty$ when $p^{\prime} \geq \frac{n}{r}$, which in particular is always the case if $n=1$.

We now introduce the index $q_{\mathbf{s}}(p)$. Let $p_{\sharp} \doteq \min \left\{p, p^{\prime}\right\}$; then, we define

$$
q_{\mathbf{s}}(p) \doteq p_{\sharp} \min _{j=1, \ldots, r}\left(1+\frac{s_{j}-(j-1) \frac{d}{2}}{\frac{d}{2}(r-j)}\right) .
$$

For $\mathbf{s}>\mathbf{g}_{\mathbf{0}}$ we have that $1<q_{\mathbf{s}}(p)<Q_{\mathbf{s}}(p)$, as it can be seen by comparing at same $j$ 's. Moreover, the requirement $q<q_{\mathbf{s}}(p)$ can be equivalently stated in terms of $\mathbf{s}$ as

$$
\mathbf{s}>\mathbf{g}_{\mathbf{0}}+\left(\frac{q}{p_{\sharp}}-1\right)_{+} \mathbf{g}_{0}^{*} .
$$

In case $\mathbf{s}=(\nu, \ldots, \nu)$, condition (4.20) then simplifies to

$$
\nu>\frac{n}{r}-1 \quad \text { and } \quad q<p_{\sharp}\left(1+\frac{\nu}{\frac{n}{r}-1}\right),
$$

so that the next theorem actually extends the result in $[\mathbf{2}, 1.8]$, at least for $p, q \neq 1$ :

Theorem 4.8. Let $p, q$, s satisfy condition (4.21) for some $1<p, q<$ $+\infty$. Then, $\mathcal{E}$ is an isomorphism of $B_{\mathbf{s}}^{p, q}$ onto $\mathcal{A}_{\mathbf{s}}^{p, q}$. 
Proof: By Corollary 4.7 we only need to prove a bound below on the norm of $\mathcal{E}^{-1}$, which amounts to the estimate

$$
\|\mathcal{E} f\|_{\mathcal{A}_{\mathbf{s}}^{p, q}} \preceq\|f\|_{B_{\mathbf{s}}^{p, q},}, \quad \forall f \in \mathcal{D}_{\Omega} .
$$

The crucial step is the following elementary lemma:

Lemma $4.9([\mathbf{2}, 4.8])$. Let $1 \leq p \leq+\infty, 1 \leq v \leq p_{\sharp}$. Then, the linear operator

$$
\left(f_{j}\right)_{j \in \mathbb{N}} \in \ell^{v}\left(L^{p}(V, d x)\right) \longmapsto \sum_{j \in \mathbb{N}} f_{j} * \chi_{j} \in L^{p}(V, d x)
$$

is bounded.

Thus, applying the lemma above with $f_{j} \doteq f * \mathcal{F}^{-1}\left(e^{-(y \mid \cdot)} \widehat{\theta}_{j}\right)$, and by Young inequality, we can bound

$$
\begin{aligned}
\left\|(\mathcal{E} f)_{y}\right\|_{p} & =\left\|\mathcal{F}^{-1}\left(e^{-(y \mid \cdot)} \widehat{f}\right)\right\|_{p}=\left\|\sum_{k \in \mathbb{N}} \mathcal{F}^{-1}\left(e^{-(y \mid \cdot)} \widehat{f} \widehat{\chi}_{k}\right)\right\|_{p} \\
& \preceq\left(\sum_{k \in \mathbb{N}}\left\|f * \chi_{k}\right\|_{p}^{p_{\sharp}}\left\|\mathcal{F}^{-1}\left(e^{-(y \mid \cdot)} \widehat{\theta}_{k}\right)\right\|_{1}^{p_{\sharp}}\right)^{\frac{1}{p_{\sharp}}} .
\end{aligned}
$$

Moreover, there exists $\gamma>0$ such that $\left\|\mathcal{F}^{-1}\left(e^{-(y \mid \cdot)} \widehat{\theta}_{k}\right)\right\|_{1} \preceq e^{-\gamma\left(y \mid \xi_{k}\right)}$ independently of $y$ in $\Omega$, and consequently

$$
\|\mathcal{E} f\|_{\mathcal{A}_{\mathbf{s}}^{p, q}}^{q} \preceq \int_{\Omega}\left(\sum_{k \in \mathbb{N}}\left\|f * \chi_{k}\right\|_{p}^{p_{\sharp}} e^{-\left(y \mid \xi_{k}\right)}\right)^{\frac{q}{p_{\sharp}}} \Delta_{\mathbf{S}}(y) \frac{d y}{\Delta(y)^{\frac{n}{r}}} .
$$

Now, if $q \leq p_{\sharp}$ we majorize the $\ell^{p_{\sharp} / q}$ norm of the integrand in the righthand side of (4.22) with its $\ell^{1}$ norm, finding as claimed that

$$
\|\mathcal{E} f\|_{\mathcal{A}_{\mathbf{s}}^{p, q}}^{q} \preceq \sum_{k \in \mathbb{N}}\left\|f * \chi_{k}\right\|_{p}^{q} \int_{\Omega} e^{-\left(y \mid \xi_{k}\right)} \Delta_{\mathbf{s}}(y) \frac{d y}{\Delta(y)^{\frac{n}{r}}}=\Gamma_{\Omega}(\mathbf{s})\|f\|_{B_{\mathbf{s}}^{p, q}}^{q},
$$

by (2.3). Otherwise, we set $u \doteq \frac{q}{p_{\sharp}}$, so that $u>1$. Then, an application of Hölder inequality in (4.22) yields that $\|\mathcal{E} f\|_{\mathcal{A}_{\mathrm{s}}^{p, q}}^{q}$ is bounded above by

$\int_{\Omega}\left(\sum_{k \in \mathbb{N}}\left\|f * \chi_{k}\right\|_{p}^{q} \Delta_{\mathbf{t}}^{u}\left(\xi_{k}^{-1}\right) e^{-\left(y \mid \xi_{k}\right)}\right)\left(\sum_{k \in \mathbb{N}} \Delta_{-\mathbf{t}}^{u^{\prime}}\left(\xi_{k}^{-1}\right) e^{-\left(y \mid \xi_{k}\right)}\right)^{\frac{u}{u^{\prime}}} \Delta_{\mathbf{s}}(y) \frac{d y}{\Delta(y)^{\frac{n}{r}}}$.

The second sum is uniformly controlled by a scalar multiple of $\Delta_{-\mathbf{t}}^{u}(y)$ for $y$ in $\Omega$ as soon as $u^{\prime} \mathbf{t}>\mathbf{g}_{\mathbf{0}}^{*}$, by Lemma 2.1 and (2.3); thus, by (2.3) 
once more we find that

$$
\|\mathcal{E} f\|_{\mathcal{A}_{\mathbf{s}}^{p, q}}^{q} \preceq \sum_{k \in \mathbb{N}}\left\|f * \chi_{k}\right\|_{p}^{q} \Delta_{\mathbf{t}}^{u}\left(\xi_{k}^{-1}\right) \int_{\Omega} e^{-\left(y \mid \xi_{k}\right)} \Delta_{-\mathbf{t}}^{u}(y) \Delta_{\mathbf{s}}(y) \frac{d y}{\Delta(y)^{\frac{n}{r}}} \preceq\|f\|_{B_{\mathbf{s}}^{p, q}}^{q}
$$

if also $\mathbf{s}-u \mathbf{t}>\mathbf{g}_{\mathbf{0}}$. Forcing the fulfilment of the two conditions on $\mathbf{t}$ is equivalent with the requirement that

$$
\mathbf{s}-\mathbf{g}_{\mathbf{0}}>\frac{u}{u^{\prime}} \mathbf{g}_{0}^{*}=\left(\frac{q}{p_{\sharp}}-1\right) \mathbf{g}_{0}^{*} .
$$

Finally, the conditions arising from two cases can be summarized in (4.21).

\subsection{Counterexamples.}

We shall now address the following question: when is Theorem 4.8 sharp? i.e., is the condition $q<q_{\mathbf{s}}(p)$ necessary for Theorem 4.8 to hold?

On behalf of Corollary 4.7, Theorem 4.8 is equivalent with the boundedness of the Cauchy operator from $B_{\mathbf{s}}^{p, q}$ into $\mathcal{A}_{\mathbf{s}}^{p, q}$. Therefore, we shall assume that $\mathbf{s}$ is strictly bigger than $\mathbf{g}_{\mathbf{0}}, p$ belongs to $] 1,+\infty[$, $1<q<Q_{\mathbf{s}}(p)$ and that

$$
\|\mathcal{E}[S]\|_{\mathcal{A}_{\mathbf{s}}^{p, q}} \preceq\|[S]\|_{B_{\mathbf{s}}^{p, q}} \quad\left([S] \in B_{\mathbf{s}}^{p, q}\right) .
$$

First, arguing as in $[\mathbf{2}, \S 4.4]$, one can easily show that (4.23) implies the existence of a constant $A_{p}$, exclusively depending on $p$, such that the inequality

$$
\left(\sum\left|a_{j}\right|^{2}\right)^{\frac{1}{2}} \leq A_{p}\left(\sum \Delta_{\mathbf{s}}\left(\xi_{j}^{-1}\right)\left|a_{j}\right|^{q}\right)^{\frac{1}{q}}
$$

holds for any choice of finite subsets $E$ of $\left\{\xi_{j}:\left|\xi_{j}\right|<1\right\}$ and of scalars $a_{j}$. Now it's just a matter of balancing $a_{j}$ with $\Delta_{\mathbf{s}}\left(\xi_{j}^{-1}\right)$ : indeed, taking $a_{j}$ to be equal to $\Delta_{\mathbf{s}}\left(\xi_{j}^{-1}\right)^{\frac{1}{2-q}}$, we have that

$$
\left(\sum_{\xi_{j} \in E} \Delta_{\mathbf{s}}\left(\xi_{j}^{-1}\right)^{\frac{2}{2-q}}\right)^{\frac{1}{2}-\frac{1}{q}} \leq A_{p}
$$

independently of the finite subset $E$, which for $q>2$ is equivalent with

$$
\int_{\Omega \cap(\mathbf{e}-\Omega)} \Delta_{\mathbf{s}^{*}}^{*}(\xi)^{\frac{2}{q-2}} \frac{d \xi}{\Delta(\xi)^{\frac{n}{r}}}<+\infty ;
$$


by $(2.13)$, this can only happen if $\mathbf{s}^{*}>\left(\frac{q}{2}-1\right) \mathbf{g}_{\mathbf{0}}$, and in terms of $q$, only if

$$
q<2 \min _{j=1, \ldots, r}\left(1+\frac{s_{j}}{(r-j) \frac{d}{2}}\right) .
$$

The second technique we employ to produce extra necessary conditions on $q$ is based on some direct calculations which provide explicit counterexamples to the surjectivity of the operator $\mathcal{E}^{-1}$. We will get better results than in (4.24) only for $p \leq 2$, which is thus what we assume from now on.

The idea is to exhibit particular distributions in $B_{\mathbf{s}}^{p, q}$ whose Cauchy transform is not in $\mathcal{A}_{\mathbf{s}}^{p, q}$, thus contradicting (4.23). In detail, for $\mathbf{u} \in \mathbb{R}^{r}$ with $\mathbf{u}>\mathbf{g}_{\mathbf{0}}$ we let

$$
\widehat{S}_{\mathbf{u}} \doteq(2 \pi)^{n} e^{-(\mathbf{e} \mid \cdot)} \Delta_{\mathbf{u}}^{*} \Delta^{-\frac{n}{r}} 1_{\Omega}
$$

First of all, note that $\widehat{S}_{\mathbf{u}}$ is an $L^{1}$ function precisely when $\mathbf{u}>\mathbf{g}_{\mathbf{0}}$, and that in this case it can also be expressed as the $L^{1}$ series $\sum_{j \in \mathbb{N}} \widehat{S}_{\mathbf{u}} \widehat{\chi}_{j}$, by dominated convergence. Therefore, $S_{\mathbf{u}}=\Gamma_{\Omega}(\mathbf{u}) \Delta_{-\mathbf{u}^{*}}(\mathbf{e}-i \cdot)$ on $V$, and it also is the sum of the series $\sum_{j \in \mathbb{N}} S_{\mathbf{u}} * \chi_{j}$ in $C_{0}(V)$; in particular the same equality holds in $\mathcal{S}^{\prime}(V)$.

Now, set $F_{\mathbf{u}} \doteq(2 \pi)^{-n} \mathcal{L} \widehat{S}_{\mathbf{u}}$ : by $(2.3)$, we have that $F_{\mathbf{u}}$ coincides with the holomorphic function $\Gamma_{\Omega}(\mathbf{u}) \Delta_{-\mathbf{u}^{*}}(\mathbf{e}-i \cdot)$ on $T_{\Omega}$, so that $F_{\mathbf{u}}=\mathcal{E}\left[S_{\mathbf{u}}\right]$ when $S_{\mathbf{u}}$ belongs to $B_{\mathbf{s}}^{p, q}$. The usual estimates being essentially too rough, instead of trying to compute $\left\|\left[S_{\mathbf{u}}\right]\right\|_{B_{\mathrm{s}}^{p, q}}$ straight by the definition we proceed differently, and wonder if $\square^{\mathbf{t}^{*}}\left[S_{\mathbf{u}}\right]$ belongs to $B_{\mathbf{s}+q \mathbf{t}}^{p, q}$ for some $\mathbf{t} \in \mathbb{R}^{r}$ with $\mathbf{t}>0$. At a formal level, the guess is that we should be concerned with an estimate for $\left\|F_{\mathbf{u}+\mathbf{t}^{*}}\right\|_{A_{\mathbf{s}+q \mathbf{t}}^{p, q}}$, and we now show that this approach actually works. Indeed, by Corollary 2.12 and (2.12) we know that the function $F_{\mathbf{u}+\mathbf{t}^{*}}$ is in $A_{\mathbf{s}+q \mathbf{t}}^{p, q}$ if and only if

$$
\begin{aligned}
p\left(\mathbf{u}+\mathbf{t}^{*}\right)> & \mathbf{g}_{\mathbf{0}}+\left(\frac{n}{r}, \ldots, \frac{n}{r}\right) \\
& \text { and } \mathbf{s}^{*}+\mathbf{g}_{\mathbf{0}}<q\left(\mathbf{u}-\left(\frac{n}{r p}, \ldots, \frac{n}{r p}\right)\right) .
\end{aligned}
$$

In this case, by (4.12), Theorem 4.6 and the remarks above we also have that $S_{\mathbf{u}+\mathbf{t}^{*}}$ is in $B_{\mathbf{s}+q \mathbf{t}}^{p, q}$ and that $S_{\mathbf{u}+\mathbf{t}^{*}}=\mathcal{E}^{-1} F_{\mathbf{u}+\mathbf{t}^{*}}$. Moreover,

$$
\square^{-\mathbf{t}^{*}}\left[S_{\mathbf{u}+\mathbf{t}^{*}}\right]=\left[S_{\mathbf{u}}\right]
$$


because the relation $\chi_{j} * \square^{-\mathbf{t}^{*}}\left[S_{\mathbf{u}+\mathbf{t}^{*}}\right]=S_{\mathbf{u}} * \chi_{j}$ holds for every $j \in \mathbb{N}$, by (4.11). In particular, $\left[S_{\mathbf{u}}\right]$ belongs to $B_{\mathbf{s}}^{p, q}$, since $\square^{-\mathbf{t}^{*}}$ is an isomorphism. Finally, we can prevent $F_{\mathbf{u}}$ from belonging to $\mathcal{A}_{\mathbf{s}}^{p, q}$ by requiring that $\left\|\left(F_{\mathbf{u}}\right)_{\mathbf{e}}\right\|_{p}=+\infty$, which on behalf of Corollary 2.12 is equivalent to the condition

$$
p \mathbf{u} \ngtr \mathbf{g}_{\mathbf{0}}+\left(\frac{n}{r}, \ldots, \frac{n}{r}\right) .
$$

Collecting (4.25) and (4.26) altogether, one can see that the best choice (in order to bound $q$ from below) comes from fixing $j \in\{1, \ldots, r\}$ and letting

$$
u_{j} \doteq \frac{1}{p}\left(\frac{n}{r}+(j-1) \frac{d}{2}\right) .
$$

Up to completing $\mathbf{u}$ with suitably high positive values, the only condition effectively remaining is the $j$-th one of the second set of inequalities in (4.25), so that in the end we have shown that Theorem 4.8 cannot hold when

$$
q>p\left(1+\frac{s_{r+1-j}}{(j-1) \frac{d}{2}}\right) .
$$

Since this is true for every $j$ in $\{1, \ldots, r\}$, we then deduce the necessary condition

$$
q \leq p \min _{j=1, \ldots, r}\left(1+\frac{s_{j}}{(r-j) \frac{d}{2}}\right)
$$

which we recall has to hold for $1<p \leq 2$. Therefore, we can merge (4.24) and (4.27) into the statement below:

Corollary 4.10. Let $p$ in $] 1,+\infty\left[, \mathbf{s}>\mathbf{g}_{0}\right.$ and $1<q<Q_{\mathbf{s}}(p)$. Then, Theorem 4.8 can hold only if

$$
q \leq \min \{p, 2\} \min _{j=1, \ldots, r}\left(1+\frac{s_{j}}{(r-j) \frac{d}{2}}\right) \doteq \widetilde{q}_{\mathbf{s}}(p),
$$

and equality can occur only if $p<2$.

In case $\mathbf{s}=(\nu, \ldots, \nu)$, one can show that the inequality in Corollary 4.10 is strict: $q<\widetilde{q}_{\mathbf{s}}(p)$, see $[\mathbf{2}, 4.34]$. It seems reasonable to conjecture that this will also be the case for general $\mathbf{s}$.

For the rank 2 case, see Theorem 5.8 below. 


\section{Bergman projections}

In the end, the main subject under investigation turns into the boundedness of Bergman projections. That is, we consider the orthogonal projector $P_{\mathbf{s}}$ of $L_{\mathbf{s}}^{2,2}$ onto $\mathcal{A}_{\mathbf{s}}^{2,2}$ and we look for conditions on $p, q$ and $\mathbf{s}$ which allow to or prevent from extending $P_{\mathbf{s}}$ as a continuous operator from $L_{\mathbf{s}}^{p, q}$ into $\mathcal{A}_{\mathbf{s}}^{p, q}$.

It is well-known, see e.g. [7], that $\mathcal{A}_{\mathbf{s}}^{2,2}$ admits a reproducing kernel $K_{\mathbf{s}}$. Therefore,

$$
\begin{aligned}
\left(P_{\mathbf{s}} F\right)(z) & \doteq\left\langle F, K_{\mathbf{s}}(\cdot, z)\right\rangle_{L_{\mathbf{s}}^{2,2}} \\
& =\int_{T_{\Omega}} K_{\mathbf{s}}(z, w) F(w) \Delta_{\mathbf{s}-\left(\frac{n}{r}, \ldots, \frac{n}{r}\right)}(\Im m w) d w
\end{aligned}
$$

for every $z \in T_{\Omega}$ and $F \in L_{\mathbf{s}}^{2,2}$, and by standard arguments, see [4] or directly apply Theorem 2.7 and (2.3) above, one can show that

$$
K_{\mathbf{s}}(z, w)=\frac{2^{s_{1}+\cdots+s_{r}} \Gamma_{\Omega}\left(\mathbf{s}^{*}+\left(\frac{n}{r}, \ldots, \frac{n}{r}\right)\right)}{(2 \pi)^{2 n} \Gamma_{\Omega}(\mathbf{s})} \Delta_{-\mathbf{s}-\left(\frac{n}{r}, \ldots, \frac{n}{r}\right)}\left(\frac{z-\bar{w}}{i}\right) .
$$

We now come to the general problem: can $P_{\mathbf{s}}$ be extended as a bounded operator from $L_{\mathbf{s}}^{p, q}$ into itself? That is, does the inequality

$$
\left\|P_{\mathbf{s}} F\right\|_{L_{\mathbf{s}}^{p, q}} \leq C\|F\|_{L_{\mathbf{s}}^{p, q}}
$$

hold for some $C>0$ and every $F \in L_{\mathbf{s}}^{p, q} \cap L_{\mathbf{s}}^{2,2}$ ?

Following [2, 4.23], we start by showing that the question is wellposed only in a restricted range of the indices involved. Indeed, note that boundedness of $P_{\mathbf{s}}$ on $L_{\mathbf{s}}^{p, q}$ implies that $P_{\mathbf{s}}\left(L_{\mathbf{s}}^{p, q}\right)=\mathcal{A}_{\mathbf{s}}^{p, q}$, at least for finite $q$, by the density result in Proposition 2.14, so that in particular the linear functional

$$
F \in L_{\mathbf{s}}^{p, q} \longmapsto\left(P_{\mathbf{s}} F\right)(i \mathbf{e})
$$

would have to belong to $\left(L_{\mathbf{s}}^{p, q}\right)^{*}$, by $(2.5)$. If in addition we assume that $1<p<+\infty$, then $[\mathbf{9}, \mathrm{V} .1 .3]$ and $(5.1)$ entail that $\Delta_{-\mathbf{s}-\left(\frac{n}{r}, \ldots, \frac{n}{r}\right)}(\mathbf{e}-i \cdot)$ is an element of $L_{\mathbf{s}}^{p^{\prime}}, q^{\prime}$, which according to the norm computations carried out in Corollary 2.12 and (2.12) is equivalent to the conditions

$$
q<Q_{\mathbf{s}}(p) \text { and } p\left(\mathbf{s}-\mathbf{g}_{\mathbf{0}}^{*}\right)>-\mathbf{g}_{\mathbf{0}}^{*}-\left(\frac{n}{r}, \ldots, \frac{n}{r}\right),
$$

once $q$ is also taken not to be 1 .

Conversely, note also that $P_{\mathbf{s}}$ is meaningfully defined on $L_{\mathbf{s}}^{p, q}$ by equation (5.1) under conditions $(5.4)$, for then $K_{\mathbf{s}}(\cdot, z)$ is in $\mathcal{A}_{\mathbf{s}}^{p^{\prime}}, q^{\prime}$ for every $z \in T_{\Omega}$. 
This settled, we look for positive answers to (5.3). The approach is slightly different and more direct than in [2]: we try to write $P_{\mathbf{s}}$ as the composite of the Cauchy extension $\mathcal{E}$ and an operator $\omega_{\mathbf{s}}$ giving a Besov-valued multiplier expression for $P_{\mathbf{s}}$. The hint comes from (5.1) and the boundary-value formula for $\mathcal{A}_{\mathrm{s}}^{p, q}$ functions stated in Corollary 4.7, though we need to extend its validity to all of $L_{\mathbf{s}}^{p, q}$. That is, we want to define a bilinear map

$$
\widetilde{\omega}_{\mathbf{s}}: \mathcal{D}_{\Omega} \times L_{\mathbf{s}}^{p, q} \longrightarrow B_{\mathbf{s}}^{p, q}
$$

patterned after the association $F \in \mathcal{A}_{\mathbf{s}}^{p, q} \mapsto F_{0}$ introduced there.

This can be achieved by letting

$$
\widetilde{\omega}_{\mathbf{s}}(\psi, G) \doteq \frac{2^{s_{1}+\cdots+s_{r}}}{\Gamma_{\Omega}(\mathbf{s})} \int_{T^{*}} G_{\tau^{*}-1} \mathbf{e} * \psi_{\tau} d \tau .
$$

Indeed, strong measurability of $G$ is used to give a distributional meaning to the integral, and then we can go along exactly the same as in the proof of (4.16) up to show an inequality of this kind:

$$
\left\|\widetilde{\omega}_{\mathbf{s}}(\psi, G)\right\|_{B_{\mathbf{s}}^{p, q}} \preceq_{\psi}\|G\|_{L_{\mathbf{s}}^{p, q}}
$$

In particular, we intend to obtain an a priori estimate for an appropriate sequence of test-functions $\phi_{N}$ : that is, we let

$$
\phi_{N} \doteq \frac{1}{(2 \pi)^{n}} \sum_{j=0}^{N} \mathcal{F}^{-1}\left(\widehat{\chi}_{j} \Delta_{\mathbf{s}^{*}}^{*} e^{-(\cdot \cdot \mathbf{e})}\right)
$$

for every $N \in \mathbb{N}$, and the claim is that there exists some positive constant $C$ such that

$$
\left\|\widetilde{\omega}_{\mathbf{s}}\left(\phi_{N}, G\right)\right\|_{B_{\mathbf{s}}^{2,2}} \leq C\|G\|_{L_{\mathbf{s}}^{2,2}}
$$

for every $G$ in $L_{\mathbf{s}}^{p, q}$ and independently of $N$ in $\mathbb{N}$.

The reason why we are interested in this choice of $\phi_{N}$ is the following: assume that (5.5) has been proved. Then, dominated convergence and reflexivity imply that $\widetilde{\omega}_{\mathbf{s}}\left(\phi_{N}, G\right)$ converges in $B_{\mathbf{s}}^{2,2}$ to a distribution $\omega_{\mathbf{s}}(G)$ which satisfies

$$
\left\|\omega_{\mathbf{s}}(G)\right\|_{B_{\mathbf{s}}^{2,2}} \leq C\|G\|_{L_{\mathbf{s}}^{2,2}}
$$

and whose duality pairing with $f$ in $\mathcal{D}_{\Omega} \hookrightarrow B_{-\mathbf{s}}^{2,2}$ is given by the formula

$$
\left.\frac{2^{s_{1}+\cdots+s_{r}}}{(2 \pi)^{n} \Gamma_{\Omega}(\mathbf{s})} \int_{T^{*}}\left\langle G_{\tau^{*}-1}, \mathcal{F}^{-1}\left(\widehat{f} \Delta_{\mathbf{s}^{*}}^{*} e^{-\left(\tau^{*}-1\right.} \mathbf{e} \mid \cdot\right)\right)^{`}\right\rangle \Delta_{\mathbf{s}}\left((\tau \mathbf{e})^{-1}\right) d \tau .
$$

But (5.5) follows from Proposition 3.14 and (4.16) once we show that

$$
\int_{T^{*}}\left\|h *\left(\phi_{N}\right)_{\tau}\right\|_{2}^{2} \Delta_{-\mathbf{s}}\left((\tau \mathbf{e})^{-1}\right) d \tau \preceq\|h\|_{B_{-\mathbf{s}}^{2,2}}^{2}
$$


In turn, Lemma 4.9 (which simply amounts to the finite intersection property of Whitney lattices in this case) and Lemma 2.1 can be used in order to bound the LHS of (5.7) with

$$
\begin{aligned}
& C \sum_{j=0}^{N} \int_{T^{*}}\left\|\mathcal{F}^{-1}\left(e^{-(\mathbf{e} \mid \cdot)} \Delta_{\mathbf{s}^{*}}^{*} \widehat{\chi}_{j} \widehat{h_{\tau^{-1}}}\right)\right\|_{2}^{2} \Delta_{-\mathbf{s}}\left((\tau \mathbf{e})^{-1}\right) \operatorname{Det} \tau d \tau \\
& \preceq \sum_{j=0}^{N} e^{-\gamma\left(\mathbf{e} \mid \xi_{j}\right)} \Delta_{2 \mathbf{s}^{*}}^{*}\left(\xi_{j}\right) \int_{T^{*}}\left\|h_{\tau^{-1}} * \chi_{j}\right\|_{2}^{2} \Delta_{-\mathbf{s}}\left((\tau \mathbf{e})^{-1}\right) \operatorname{Det} \tau d \tau \\
& =\sum_{j=0}^{N} e^{-\gamma\left(\mathbf{e} \mid \xi_{j}\right)} \Delta_{2 \mathbf{s}^{*}}^{*}\left(\xi_{j}\right) \int_{T^{*}}\left\|h * \chi_{\tau \tau_{j}}\right\|_{2}^{2} \Delta_{-\mathbf{s}}\left((\tau \mathbf{e})^{-1}\right) d \tau \doteq A_{N} .
\end{aligned}
$$

Now, by definition of the modular function $\Delta_{T^{*}}$ we have

$$
\int_{T^{*}}\left\|h * \chi_{\tau \tau_{j}}\right\|_{2}^{2} \Delta_{-\mathbf{s}}\left((\tau \mathbf{e})^{-1}\right) d \tau=\Delta_{T^{*}}^{-1}\left(\tau_{j}\right) \Delta_{-\mathbf{s}^{*}}^{*}\left(\xi_{j}\right)\|h\|_{B_{-\mathbf{s}}^{2,2}}^{2},
$$

and therefore, by (3.8) and Lemma 2.1 we can conclude that

$$
A_{N}=\sum_{j=0}^{N} e^{-\gamma\left(\mathbf{e} \mid \xi_{j}\right)} \Delta_{\mathbf{s}^{*}-\mathbf{g}_{\mathbf{0}}^{*}+\mathbf{g}_{\mathbf{0}}}^{*}\left(\xi_{j}\right)\|h\|_{B_{-\mathbf{s}}^{2,2}}^{2} \preceq \Gamma_{\Omega}\left(\mathbf{s}^{*}-\mathbf{g}_{\mathbf{0}}^{*}+\mathbf{g}_{\mathbf{0}}\right)\|h\|_{B_{-\mathbf{s}}^{2,2}}^{2}
$$

as soon as $\mathbf{s}>\mathbf{g}_{\mathbf{0}}$, so that (5.7) and hence (5.5) finally follow.

Moreover, we are now also able to prove that $\mathcal{E}$ actually is the adjoint of $\omega_{\mathbf{s}}$, in the Hilbert space sense. To avoid confusion, we will name $\widetilde{\mathcal{E}}$ the composite of $\mathcal{E}$ and the inclusion $\mathcal{A}_{\mathbf{s}}^{2,2} \hookrightarrow L_{\mathbf{s}}^{2,2}$.

So, first of all, we have to define an inner product $\langle\langle\cdot, \cdot\rangle\rangle_{\mathrm{s}}$ underlying the \|\|$_{B_{\mathrm{s}}^{2,2}}$ norm. For $\mathbf{s}>\mathbf{g}_{\mathbf{0}}$ this can be easily accomplished by combining the results of Theorems 2.7 and 4.8: indeed, they show that

$$
B_{\mathbf{s}}^{2,2}=\mathcal{F}^{-1}\left(L_{\mathbf{s}}^{2}(\Omega)\right),
$$

and thus we can let

$$
\langle\langle f, g\rangle\rangle_{\mathbf{s}} \doteq \frac{\Gamma_{\Omega}(\mathbf{s})}{2^{s_{1}+\cdots+s_{r}}} \int_{\Omega} \widehat{f}(\xi) \overline{\widehat{g}(\xi)} \Delta_{\mathbf{s}}\left(\xi^{-1}\right) d \xi
$$

for $f, g \in \mathcal{D}_{\Omega}$ (or even $\widehat{f}, \widehat{g}$ in $L_{\mathbf{s}}^{2}(\Omega)$ ).

On the other hand, $B_{\mathbf{s}}^{2,2}$ and $B_{-\mathbf{s}}^{2,2}$ are isomorphic as Banach spaces by means of the duality pairing (3.18), and we shall need to make use of the following link existing between the two representations of $B_{\mathbf{s}}^{2,2}$ : if $g^{*} \doteq \bar{g}$ denotes the usual involution on $L^{2}(V, d x)$, then

$$
\square^{-\mathbf{s}^{*}}: B_{\mathbf{s}}^{2,2} \longrightarrow B_{-\mathbf{s}}^{2,2}
$$


sets up an isomorphism for which the relation

$$
\int_{\Omega} \widehat{f}(\xi) \overline{\widehat{g}(\xi)} \Delta_{\mathbf{s}}\left(\xi^{-1}\right) d \xi={ }_{B_{\mathbf{s}}, 2}\left\langle f, \square^{-\mathbf{s}^{*}} g^{*}\right\rangle_{B_{-\mathbf{s}}^{2,2}}
$$

holds, for every $f, g \in \mathcal{D}_{\Omega}$.

Finally, as a consequence of (5.6), we are ready to show that $\widetilde{\mathcal{E}}^{*}=\omega_{\mathbf{s}}$, i.e.

$$
\left\langle\left\langle f, \omega_{\mathbf{s}}(G)\right\rangle\right\rangle_{\mathbf{s}}=\langle\mathcal{E} f, G\rangle_{L_{\mathbf{s}}^{2,2}}
$$

for every $f \in \mathcal{D}_{\Omega}, G \in L_{\mathbf{s}}^{2,2}$. For then, owing to (5.8) and (5.6) we have

$$
\begin{aligned}
\left\langle\left\langle f, \omega_{\mathbf{s}}(G)\right\rangle\right\rangle_{\mathbf{s}} & =\frac{\Gamma_{\Omega}(\mathbf{s})}{2^{s_{1}+\cdots+s_{r}}} B_{\mathbf{s}}^{2,2}\left\langle f, \square^{-\mathbf{s}^{*}}\left(\omega_{\mathbf{s}} G\right)^{*}\right\rangle_{B_{-\mathbf{s}}^{2,2}} \\
& =\int_{\Omega}\left\langle e^{-(y \mid \cdot)} \widehat{f}, 1_{\Omega} \widehat{G}_{y}\right\rangle_{L^{2}(\Omega, d \xi)} \Delta_{\mathbf{s}}(y) \frac{d y}{\Delta(y)^{\frac{n}{r}}} \\
& =\int_{\Omega}\left\langle(\mathcal{E} f)_{y}, G_{y}\right\rangle_{L^{2}(V, d x)} \Delta_{\mathbf{s}}(y) \frac{d y}{\Delta(y)^{\frac{n}{r}}}=\langle\mathcal{E} f, G\rangle_{L_{\mathbf{s}}^{2,2}} .
\end{aligned}
$$

Thus, we have proved the first half of the following:

Proposition 5.1. Assume $\mathbf{s}>\mathbf{g}_{\mathbf{0}}$. Then, $\widetilde{\mathcal{E}}$ is the adjoint of $\omega_{\mathbf{s}}$. Moreover, $P_{\mathbf{s}}$ factors as $\widetilde{\mathcal{E}} \circ \omega_{\mathbf{s}}$ on $L_{\mathbf{s}}^{2,2}$.

Proof: Set $R_{\mathrm{s}} \doteq \widetilde{\mathcal{E}} \circ \omega_{\mathrm{s}}$. We claim that $R_{\mathrm{s}}$ is the identity on $\mathcal{A}_{\mathrm{s}}^{2,2}$. This is equivalent to the last statement of the proposition, for then $R_{\mathrm{s}}$ is the orthogonal projector onto $\mathcal{A}_{\mathbf{s}}^{2,2}$, by (5.9) and the claim, and thus it coincides with $P_{\mathbf{s}}$. Now, $\omega_{\mathbf{s}}(G)$ has been defined as the limit of $\widetilde{\omega}_{\mathbf{s}}\left(\phi_{N}, G\right)$; in particular, for $G \in \mathcal{A}_{\mathbf{s}}^{2,2}$ we already know that

$$
\frac{2^{-s_{1}-\cdots-s_{r}} \Gamma_{\Omega}(\mathbf{s})}{a\left(\phi_{N}\right)} \mathcal{E}\left(\widetilde{\omega}_{\mathbf{s}}\left(\phi_{N}, G\right)\right)=G
$$

for every $N \in \mathbb{N}$, by Corollary 4.7. Since $a\left(\phi_{N}\right) \rightarrow_{N} 2^{-s_{1}-\cdots-s_{r}} \Gamma_{\Omega}(\mathbf{s})$, we are done.

It is now easy to extend Proposition 5.1 to general Besov spaces, even though some attention has to be paid to define the right duality pairings.

So, we first extend the involution on $L^{2}(V, d x)$ to functions on the tube $T_{\Omega}$ by letting

$$
G^{*}(w) \doteq \overline{G(-\bar{w})}
$$

(note that holomorphic functions are preserved), and then for $\{p, q\} \neq\{2\}$ we define the following pairing between $L_{\mathbf{s}}^{p, q}$ and $L_{\mathbf{s}}^{p^{\prime}, q^{\prime}}$ :

$$
L_{\mathbf{s}}^{p^{\prime}, q^{\prime}}\langle F, G\rangle_{L_{\mathbf{s}}^{p, q}} \doteq \int_{T_{\Omega}} F(w) G(-\bar{w}) \Delta_{\mathbf{s}-\left(\frac{n}{r}, \ldots, \frac{n}{r}\right)}(\Im m w) d w .
$$


Finally, we introduce the operator $\pi_{\mathbf{s}} \doteq \frac{\Gamma_{\Omega}(\mathbf{s})}{2^{s_{1}+\cdots+s_{r}}} \square^{-\mathbf{s}^{*}}{ }^{*} \omega_{\mathbf{s}}$, which according to (5.6) is given for $G$ in $L_{\mathbf{s}}^{2,2}$ and $f$ in $\mathcal{D}_{\Omega} \hookrightarrow B_{\mathbf{s}}^{2,2}$ by the equation

$$
{ }_{B_{-\mathbf{s}}^{2,2}}\left\langle\pi_{\mathbf{s}}(G), f\right\rangle_{B_{\mathbf{s}}^{2,2}}=\int_{\Omega}\left(\int_{\Omega} e^{-(y \mid \xi)} \widehat{G}_{y}(\xi) \widehat{f}(\xi) d \xi\right) \Delta_{\mathbf{s}}(y) \frac{d y}{\Delta(y)^{\frac{n}{r}}} .
$$

Corollary 5.2. Let $p, q$ belong to $] 1,+\infty\left[\right.$ and assume that $\mathbf{s}>\mathbf{g}_{0}$. Then, the following facts are equivalent:

i) $\widetilde{\mathcal{E}}$ is bounded from $B_{\mathbf{s}}^{p, q}$ into $L_{\mathbf{s}}^{p, q}$.

ii) $\pi_{\mathbf{s}}$ is bounded from $L_{\mathbf{s}}^{p^{\prime}, q^{\prime}}$ into $B_{-\left(q^{\prime}-1\right) \mathbf{s}}^{p^{\prime}, q^{\prime}}$.

iii) $\omega_{\mathbf{s}}$ is bounded from $L_{\mathbf{s}}^{p^{\prime}}, q^{\prime}$ into $B_{\mathbf{s}}^{p^{\prime}}, q^{\prime}$.

In particular, if i) holds, $\pi_{\mathbf{s}}$ is the dual operator of $\widetilde{\mathcal{E}}$.

Proof: Since $\square^{-\mathbf{s}^{*}}$ is an isomorphism, ii) $\Leftrightarrow$ iii) is trivial, while owing to $(5.8)$ and $(5.9)$ i) $\Leftrightarrow$ ii) follows from the chain of equalities

$$
\begin{aligned}
& L_{\mathbf{s}}^{p^{\prime}, q^{\prime}}\langle G, \mathcal{E} f\rangle_{L_{\mathbf{s}}^{p, q}}=\left\langle\mathcal{E} f, G^{*}\right\rangle_{L_{\mathbf{s}}^{2,2}} \\
& =\left\langle\left\langle f, \omega_{\mathbf{s}}\left(G^{*}\right)\right\rangle\right\rangle_{\mathbf{s}} \\
& ={ }_{B_{-\left(q^{\prime}-1\right) \mathbf{s}}^{p^{\prime}, q^{\prime}}}\left\langle\pi_{\mathbf{s}}(G), f\right\rangle_{B_{\mathbf{s}}^{p, q}},
\end{aligned}
$$

which is true for every $G$ in $L_{\mathbf{s}}^{2,2} \cap L_{\mathbf{s}}^{p^{\prime}, q^{\prime}}$ and $f$ in $\mathcal{D}_{\Omega} \hookrightarrow B_{\mathbf{s}}^{p, q} \cap B_{\mathbf{s}}^{2,2}$. In particular, this shows that $\pi_{\mathbf{s}}$ is the (Banach-wise) adjoint of $\widetilde{\mathcal{E}}$ whenever one of them, and hence both, is bounded.

Thus, by Theorem 4.8 we also obtain the following partial result concerning $\omega_{\mathbf{s}}$ :

Proposition 5.3. Let $1<p<+\infty, \mathbf{s}>\mathbf{g}_{0}$ and $q_{\mathbf{s}}^{\prime}(p)<q<+\infty$. Then, the linear operator $\omega_{\mathbf{s}}$ defined in (5.6) is bounded from $L_{\mathbf{s}}^{p, q}$ into $B_{\mathbf{s}}^{p, q}$.

Remark 5.4. $P_{\mathbf{s}}$ is bounded on $L_{\mathbf{s}}^{p, q}$ if and only if it is bounded on $L_{\mathbf{s}}^{p^{\prime}, q^{\prime}}$.

Indeed, a reproducing kernel is hermitian, so that by (5.2) we have that

$$
K_{\mathbf{S}}(z, w)=K_{\mathbf{s}}(-\bar{w},-\bar{z})=\overline{K_{\mathbf{s}}(-\bar{z},-\bar{w})}
$$

In particular, $P_{\mathbf{S}}\left(G^{*}\right)=\left(P_{\mathbf{S}} G\right)^{*}$ for $G$ in $L_{\mathbf{s}}^{2,2}$, and therefore

$$
\begin{aligned}
L_{\mathbf{s}}^{p^{\prime}, q^{\prime}}\left\langle G, P_{\mathbf{s}} F\right\rangle_{L_{\mathbf{s}}^{p, q}} & =\left\langle P_{\mathbf{s}} F, G^{*}\right\rangle_{L_{\mathbf{s}}^{2,2}} \\
& =\left\langle F,\left(P_{\mathbf{S}} G\right)^{*}\right\rangle_{L_{\mathbf{s}}^{2,2}}={ }_{L_{\mathbf{s}}^{p^{\prime}, q}}\left\langle P_{\mathbf{S}} G, F\right\rangle_{L_{\mathbf{s}}^{p, q}}
\end{aligned}
$$

if $G$ also belongs to $L_{\mathbf{s}}^{p^{\prime}}, q^{\prime}$ and $F$ is in $L_{\mathbf{s}}^{2,2} \cap L_{\mathbf{s}}^{p, q}$.

As a consequence, the dual conditions to those in (5.4) must hold as well whenever $P_{\mathbf{s}}$ is bounded on $L_{\mathbf{s}}^{p, q}$. 
The final goal is to show that the main problems encountered so far - boundedness of Bergman projections and the conclusion of Theorem 4.8 - are in fact equivalent, even though at present a new condition on $\mathbf{s}$ is needed in order to get a full result. This will also provide a partial answer to (5.3).

But first, we briefly investigate the dual of a Bergman space: besides being important on its own, this issue will also lead to state another property of Bergman spaces which will be shown equivalent to those mentioned above.

Lemma 5.5. Let $\mathbf{s}>\mathbf{g}_{\mathbf{0}}, 1<p, q<+\infty$ and assume that conditions (5.4) hold. Then, the map

$$
j: \mathcal{A}_{\mathbf{s}}^{p, q} \hookrightarrow\left(\mathcal{A}_{\mathbf{s}}^{p^{\prime}, q^{\prime}}\right)^{*}
$$

is an embedding (w.r.t. the duality pairing induced by ${ }_{L_{\mathbf{s}}^{p^{\prime}, q^{\prime}}}\langle\cdot, \cdot\rangle_{L_{\mathbf{s}}^{p, q}}$ ).

Proof: We only show that $j$ is injective, the other verifications being trivial. So, let $F$ belong to $\operatorname{ker} j$. By Proposition 2.14, we can choose a sequence $\left(F_{m}\right)_{m \in \mathbb{N}}$ in $\mathcal{A}_{\mathbf{s}}^{p, q} \cap \mathcal{A}_{\mathbf{s}}^{2,2}$ converging to $F$ in $\mathcal{A}_{\mathbf{s}}^{p, q}$. Moreover, $K_{\mathbf{s}}(\cdot, z)$ is in $\mathcal{A}_{\mathbf{s}}^{p^{\prime}}, q^{\prime}$ for every $z \in T_{\Omega}$, by (5.4). Therefore,

$$
\begin{aligned}
0=j(F)\left(K_{\mathbf{s}}(\cdot, z)\right) & ={ }_{L_{\mathbf{s}}^{p^{\prime}, q^{\prime}}}\left\langle K_{\mathbf{s}}(\cdot, z), F\right\rangle_{L_{\mathbf{s}}^{p, q}} \\
& =\lim _{m} L_{L_{\mathbf{s}}^{p^{\prime}, q}}\left\langle K_{\mathbf{s}}(\cdot, z), F_{m}\right\rangle_{L_{\mathbf{s}}^{p, q}} \\
& =\lim _{m} \overline{\left\langle F_{m}^{*}, K_{\mathbf{s}}(\cdot, z)\right\rangle_{\mathcal{A}_{\mathbf{s}}^{2,2}}} \\
& =\lim _{m} F_{m}(-\bar{z})=F(-\bar{z}),
\end{aligned}
$$

by the reproducing property of $K_{\mathbf{s}}$ and Proposition 2.3. Since $z$ is arbitrary, we are done.

For convenience sake, we restate the second condition in (5.4) focusing on $p$ : letting

$$
p_{\mathbf{s}} \doteq 1+\min _{j=1, \ldots, r} \frac{s_{j}+\frac{n}{r}}{\left((r-j) \frac{d}{2}-s_{j}\right)_{+}},
$$

we have that

$$
p\left(\mathbf{s}-\mathbf{g}_{\mathbf{0}}^{*}\right)>-\mathbf{g}_{\mathbf{0}}^{*}-\left(\frac{n}{r}, \ldots, \frac{n}{r}\right) \Longleftrightarrow p<p_{\mathbf{s}} .
$$

Note that $p_{\mathbf{s}}>2$, and that $p_{\mathbf{s}}=+\infty$ if and only if $\mathbf{s} \geq \mathbf{g}_{\mathbf{0}}^{*}$. 
Theorem 5.6. Let $\mathbf{s}>\mathbf{g}_{\mathbf{0}}$, and assume that $1<p<p_{\mathbf{s}}, q_{\mathbf{s}}^{\prime}(p)<q<Q_{\mathbf{s}}(p)$. Then, the following properties are equivalent:

1) $P_{\mathbf{s}}$ admits a bounded extension from $L_{\mathbf{s}}^{p, q}$ onto $\mathcal{A}_{\mathbf{s}}^{p, q}$.

2) $\omega_{\mathbf{s}}$ admits a bounded extension from $L_{\mathbf{s}}^{p^{\prime}, q^{\prime}}$ onto $B_{\mathbf{s}}^{p^{\prime}}, q^{\prime}$.

3) $\mathcal{E}$ is an isomorphism from $B_{\mathbf{s}}^{p, q}$ onto $\mathcal{A}_{\mathbf{s}}^{p, q}$.

4) $j$ is an isomorphism from $\mathcal{A}_{\mathbf{s}}^{p, q}$ onto $\left(\mathcal{A}_{\mathbf{s}}^{p^{\prime}, q^{\prime}}\right)^{*}$.

Proof: We start with a preliminary remark: under the stated hypotheses, Theorem 4.8 applies w.r.t. $p^{\prime}, q^{\prime}$ and $\mathbf{s}$, so that

$$
\mathcal{E} \text { is an isomorphism from } B_{\mathbf{s}}^{p^{\prime}, q^{\prime}} \text { onto } \mathcal{A}_{\mathbf{s}}^{p^{\prime}, q^{\prime}} \text {. }
$$

$1) \Rightarrow 2)$ : As a consequence of Remark 5.4 and $(5.13), \mathcal{E}^{-1} \circ P_{\mathbf{s}}$ is bounded from $L_{\mathbf{s}}^{p^{\prime}, q^{\prime}}$ onto $B_{\mathbf{s}}^{p^{\prime}}, q^{\prime}$, and by Proposition 5.1 it equals $\omega_{\mathbf{s}}$ on $L_{\mathbf{s}}^{2,2} \cap L_{\mathbf{s}}^{p^{\prime}, q^{\prime}}$.

$2) \Rightarrow 3$ ): This is part of Corollary 5.2 , since the image of $\widetilde{\mathcal{E}}$ is then clearly contained in $\mathcal{A}_{\mathrm{s}}^{p, q}$; surjectivity of $\mathcal{E}$ now follows from Corollary 4.7.

$3) \Rightarrow 1$ ): According to Proposition 5.3, $\omega_{\mathbf{s}}$ is bounded from $L_{\mathbf{s}}^{p, q}$ into $B_{\mathrm{s}}^{p, q}$. Thus, $\mathcal{E} \circ \omega_{\mathbf{s}}$ is bounded from $L_{\mathbf{s}}^{p, q}$ into $\mathcal{A}_{\mathbf{s}}^{p, q}$, and it is equal to $P_{\mathbf{s}}$ on $L_{\mathbf{s}}^{2,2} \cap L_{\mathbf{s}}^{p, q}$ by Proposition 5.1 ; surjectivity of $P_{\mathbf{s}}$ finally follows from the density result of Proposition 2.14.

$3) \Rightarrow 4)$ : It is sufficient to show that $j$ is surjective. So, take $\Phi \in$ $\left(\mathcal{A}_{\mathrm{s}}^{p^{\prime}, q^{\prime}}\right)^{*}$. By (5.13), $\Psi \doteq \Phi \circ \mathcal{E}$ belongs to $\left(B_{\mathrm{s}}^{p^{\prime}, q^{\prime}}\right)^{*}$. In particular, owing to Proposition 3.14, there exists $[U] \in B_{-(q-1) \text { s }}^{p, q}$ representing $\Psi$. Then, $[X] \doteq \square^{\mathbf{s}^{*}}[U] \in B_{\mathbf{s}}^{p, q}$, so that $F \doteq \frac{2^{s_{1}+\cdots+s_{r}}}{\Gamma_{\Omega}(\mathbf{s})} \mathcal{E}[X]$ belongs to $\mathcal{A}_{\mathbf{s}}^{p, q}$. But $\mathcal{E} \circ \omega_{\mathbf{s}}$ is the identity on $\mathcal{A}_{\mathbf{s}}^{p^{\prime}}, q^{\prime}$, by Proposition 5.1 , so that by the adjunction (5.11) we have that for every $G \in L_{\mathbf{s}}^{p^{\prime}}, q^{\prime}$

$$
\begin{aligned}
\Phi(G) & =\Psi\left(\mathcal{E}^{-1} G\right)={ }_{B_{\mathbf{s}}^{p^{\prime}, q^{\prime}}}\left\langle\mathcal{E}^{-1} G,[U]\right\rangle_{B_{(1-q) \mathbf{s}}^{p, q}} \\
& ={ }_{B_{\mathbf{s}}^{p^{\prime}, q^{\prime}}}\left\langle\omega_{\mathbf{s}} G,[U]\right\rangle_{B_{(1-q) \mathbf{s}}^{p, q}} \\
& =\frac{2^{s_{1}+\cdots+s_{r}}}{\Gamma_{\Omega}(\mathbf{s})}{ }_{B_{-\left(q^{\prime}-1\right) \mathbf{s}}^{p^{\prime}, q^{\prime}}}\left\langle\pi_{\mathbf{s}} G,[X]\right\rangle_{B_{\mathbf{s}}^{p, q}} \\
& ={ }_{L_{\mathbf{s}}^{p^{\prime}, q^{\prime}}}\langle G, F\rangle_{L_{\mathbf{s}}^{p, q}}=j(F)(G),
\end{aligned}
$$

i.e., $j(F)=\Phi$. 
$4) \Rightarrow 3$ ): According to Corollary 4.7, it is enough to prove a uniform estimate

$$
\|\mathcal{E} f\|_{\mathcal{A}_{\mathbf{s}}^{p, q}} \preceq\|f\|_{B_{\mathbf{s}}^{p, q}}
$$

for $f \in \mathcal{D}_{\Omega}$. But $j$ is an isomorphism, so that

$$
\|\mathcal{E} f\|_{\mathcal{A}_{\mathbf{s}}^{p, q}} \preceq \sup \left\{|j(\mathcal{E} f)(G)|:\|G\|_{\mathcal{A}_{\mathbf{s}}^{p^{\prime}, q^{\prime}}} \leq 1, G \in \mathcal{A}_{\mathbf{s}}^{2,2}\right\} .
$$

Since $\omega_{\mathbf{s}} \circ \mathcal{E}$ is the identity on $\mathcal{A}_{\mathbf{s}}^{2,2}$, owing to (5.11) we obtain that

$$
\begin{aligned}
j(\mathcal{E} f)(G) & ={ }_{B_{-\left(q^{\prime}-1\right) \mathbf{s}}^{p^{\prime}, q^{\prime}}}\left\langle\pi_{\mathbf{s}}(G), f\right\rangle_{B_{\mathbf{s}}^{p, q}} \\
& =\frac{\Gamma_{\Omega}(\mathbf{s})}{2^{s_{1}+\cdots+s_{r}}} B_{\left(1-q^{\prime}\right) \mathbf{s}}^{p^{\prime}, q^{\prime}}\left\langle\square^{-\mathbf{s}^{*}}\left(\mathcal{E}^{-1} G\right), f\right\rangle_{B_{\mathbf{s}}^{p, q}} ;
\end{aligned}
$$

$\square^{-\mathbf{s}^{*}}$ being an isomorphism, (5.13) and (5.14) give us the result.

Remark 5.7. The implications " $1 \Rightarrow 2$ ", " $2 \Rightarrow 3$ " and " $4 \Rightarrow 3$ " still continue to hold in the wider range $1<p<+\infty, 1<q^{\prime}<Q_{\mathbf{s}}\left(p^{\prime}\right)$, for they only make use of the boundedness of $\mathcal{E}^{-1}$ on $\mathcal{A}_{\mathbf{s}}^{p^{\prime}, q^{\prime}}$, and owing to Corollary 4.7 and (4.19) this happens in the range stated above.

Proof of Corollary 1.4: On behalf of Theorem 5.6,1) is a consequence of Theorem 4.8, while 2) follows from Remark 5.4 and the counterexamples in Corollary 4.10 .

\subsection{The rank 2 case.}

Finally, we briefly discuss the situation depicted by Corollary 1.4 in the special case of forward light cones, which are the prototypes for irreducible symmetric cones of rank two. So, for $n \geq 3$ let

$$
\Omega \equiv \Lambda_{n} \doteq\left\{y=\left(y^{\prime}, y_{n}\right) \in \mathbb{R}^{n-1} \times \mathbb{R}\left|y_{n}>\right| y^{\prime} \mid\right\} .
$$

Then, for an appropriate choice of the Jordan frame $\left(c_{1}, c_{2}\right)$ we have

$$
\Delta_{1}(y)=y_{n}-y_{1}, \quad \Delta(y)=y_{n}^{2}-\left|y^{\prime}\right|^{2} .
$$

Moreover, the usual assumption $\mathbf{s}>\mathbf{g}_{0}$ becomes $s_{1}>0, s_{2}>\frac{n-2}{2}$ here.

Let us remark once more that sharper results are obtained in $[\mathbf{2}, 5.11]$ for light cones when $s_{1}=s_{2}=\nu$. But in fact we shall see below that new interesting phenomena occur precisely when $s_{1} \leq \frac{n-2}{2}$, so that in particular $s_{1}$ and $s_{2}$ are forced to be different. 
Now, note that the various indices involved in Corollary 1.4 all attain their minima at $j=1$, and thus we find

$$
\begin{gathered}
p_{\mathbf{s}}=1+\frac{2 s_{1}+n}{\left(n-2-2 s_{1}\right)_{+}}=\left\{\begin{array}{ll}
\frac{2(n-1)}{n-2-2 s_{1}}, & \text { if } s_{1}<\frac{n-2}{2}, \\
+\infty, & \text { otherwise }
\end{array},\right. \\
Q_{\mathbf{s}}(p)=\frac{1+\frac{2 s_{1}}{n-2}}{\left(1-\frac{n}{(n-2) p}\right)_{+}}, \quad q_{\mathbf{s}}(p)=p_{\sharp}\left(1+\frac{2 s_{1}}{n-2}\right), \\
\widetilde{q}_{\mathbf{s}}(p)=\frac{\max \left\{2, p^{\prime}\right\}}{p^{\prime}} q_{\mathbf{s}}(p) .
\end{gathered}
$$

Moreover, one can check that $Q_{\mathbf{s}}(p)=q_{\mathbf{s}}^{\prime}(p) \Leftrightarrow p=p_{\mathbf{s}}$ when $s_{1}<\frac{n-2}{2}$, and therefore we can translate the statement of Corollary 1.4 into the following:

Theorem 5.8. Let $n \geq 3, \Omega \doteq \Lambda_{n}, 1<p, q<+\infty$ and $s_{1}>0$, $s_{2}>\frac{n-2}{2}$. Then,

1) $P_{\mathbf{s}}$ is bounded on $L_{\mathbf{s}}^{p, q}\left(T_{\Omega}\right)$ if $q_{\mathbf{s}}^{\prime}(p)<q<q_{\mathbf{s}}(p)$.

2) The gap between positive and negative results is given by the region where

$$
p \geq 2 \quad \text { and } \quad \max \left\{q_{\mathbf{s}}^{\prime}(p), q_{\mathbf{s}}(p)\right\} \leq q<\min \left\{q_{\mathbf{s}}(2), Q_{\mathbf{s}}(p)\right\}
$$

and its dual one.

And of course,

3) $P_{\mathbf{s}}$ is unbounded on $L_{\mathbf{s}}^{p, q}$ in the remaining regions.

Note that $q_{\mathbf{s}}(p)$ becomes smaller than 2 for values of $p$ close to 1 and $+\infty$ when $2 s_{1} \leq n-2$. Also, one has that $Q_{\mathbf{s}}(p)<q_{\mathbf{s}}(2)$ if and only if $p>\frac{2 n}{n-2}$, which is an effective restriction only if $s_{1}>\frac{n-2}{2 n}$.

In particular, for $p=2$ the following statement holds true without exception:

Corollary 5.9. Let $n \geq 3, \Omega \doteq \Lambda_{n}, 1<q<+\infty$ and $s_{1}>0, s_{2}>\frac{n-2}{2}$. Then, $P_{\mathbf{s}}$ is bounded on $L_{\mathbf{s}}^{2, q}\left(T_{\Omega}\right)$ if and only if $q_{\mathbf{s}}^{\prime}(2)<q<q_{\mathbf{s}}(2)$. 


\section{References}

[1] D. BéKollé, A. Bonami and G. Garrigós, Littlewood-Paley decompositions related to symmetric cones, IMHOTEP J. Afr. Math. Pures Appl. 3(1) (2000), 11-41.

[2] D. Békollé, A. Bonami, G. Garrigós and F. Ricci, Littlewood-Paley decompositions related to symmetric cones and Bergman projections in tube domains, Proc. London Math. Soc. (3) 89(2) (2004), 317-360.

[3] D. Békollé, A. Bonami, M. M. Pelooso and F. Ricci, Boundedness of Bergman projections on tube domains over light cones, Math. Z. 237(1) (2001), 31-59.

[4] D. Békollé and A. Temgoua Kagou, Reproducing properties and $L^{p}$-estimates for Bergman projections in Siegel domains of type II, Studia Math. 115(3) (1995), 219-239.

[5] A. Bonami, Three related problems of Bergman spaces of tube domains over symmetric cones, Harmonic analysis on complex homogeneous domains and Lie groups (Rome, 2001), Atti Accad. Naz. Lincei Cl. Sci. Fis. Mat. Natur. Rend. Lincei (9) Mat. Appl. 13(3-4) (2002), 183-197.

[6] R. R. Colfman and R. Rochberg, Representation theorems for holomorphic and harmonic functions in $L^{p}$, in: "Representation theorems for Hardy spaces", Astérisque 77, Soc. Math. France, Paris, 1980, pp. 11-66.

[7] J. FARAUt AND A. KorÁnyI, "Analysis on symmetric cones", Oxford Mathematical Monographs. Oxford Science Publications, The Clarendon Press, Oxford University Press, New York, 1994.

[8] M. Frazier, B. Jawerth and G. Weiss, "Littlewood-Paley theory and the study of function spaces", CBMS Regional Conference Series in Mathematics 79, Published for the Conference Board of the Mathematical Sciences, Washington, DC; by the American Mathematical Society, Providence, RI, 1991.

[9] J. García-Cuerva and J. L. Rubio de Francia, "Weighted norm inequalities and related topics", North-Holland Mathematics Studies 116, Notas de Matemática 104, North-Holland Publishing Co., Amsterdam, 1985.

[10] G. Garrigós, Generalized Hardy spaces on tube domains over cones, Colloq. Math. 90(2) (2001), 213-251.

[11] S. G. Gindikin, Analysis in homogeneous domains, (Russian), Uspehi Mat. Nauk 19(4) (118) (1964), 3-92. 
[12] S. Helgason, "Differential geometry, Lie groups, and symmetric spaces", Pure and Applied Mathematics 80, Academic Press, Inc. [Harcourt Brace Jovanovich, Publishers], New York-London, 1978.

[13] L. HörmANDER, "The analysis of linear partial differential operators. I. Distribution theory and Fourier analysis", Grundlehren der Mathematischen Wissenschaften 256, Springer-Verlag, Berlin, 1983.

[14] I. ŁABA AND T. WOLFF, A local smoothing estimate in higher dimensions, Dedicated to the memory of Tom Wolff, J. Anal. Math. 88 (2002), 149-171.

[15] F. Ricci And M. TAIBleson, Boundary values of harmonic functions in mixed norm spaces and their atomic structure, Ann. Scuola Norm. Sup. Pisa Cl. Sci. (4) 10(1) (1983), 1-54.

[16] E. M. Stein AND G. Weiss, "Introduction to Fourier analysis on Euclidean spaces", Princeton Mathematical Series 32, Princeton University Press, Princeton, N.J., 1971.

[17] H. Triebel, "Theory of function spaces", Monographs in Mathematics 78, Birkhäuser Verlag, Basel, 1983.

[18] È. B. Vinberg, S. G. Gindikin and I. I. PJateckilı-ŠApiro, Classification and canonical realization of complex homogeneous bounded domains, (Russian), Trudy Moskov. Mat. Obšč. 12 (1963), 359-388.

[19] K. Yosida, "Functional analysis", Die Grundlehren der Mathematischen Wissenschaften 123, Academic Press, Inc., New York; Springer-Verlag, Berlin, 1965.

Scuola Normale Superiore

Piazza dei Cavalieri, 7

56126 Pisa

Italy

E-mail address: debertol@sns.it

Primera versió rebuda el 4 de desembre de 2003, darrera versió rebuda el 9 de febrer de 2005 